\title{
Oxygen abundances in planet-harbouring stars ${ }^{\star} \star \star \star$ Comparison of different abundance indicators
}

\author{
A. Ecuvillon ${ }^{1}$, G. Israelian ${ }^{1}$, N. C. Santos ${ }^{2,3}$, N. G. Shchukina ${ }^{4}$, M. Mayor ${ }^{3}$, and R. Rebolo ${ }^{1,5}$ \\ 1 Instituto de Astrofísica de Canarias, 38200 La Laguna, Tenerife, Spain \\ e-mail: aecuvill@ll.iac.es \\ 2 Centro de Astronomia e Astrofisica de Universidade de Lisboa, Observatorio Astronomico de Lisboa, Tapada de Ajuda, \\ 1349-018 Lisboa, Portugal \\ 3 Observatoire de Genève, $51 \mathrm{ch}$. des Maillettes, 1290 Sauverny, Switzerland \\ ${ }^{4}$ Main Astronomical Observatory, National Academy of Sciences, 27 Zabolotnogo Street, 03680 Kyiv-127, Ukraine \\ 5 Consejo Superior de Investigaciones Científicas, Spain
}

Received 18 May 2005 / Accepted 16 August 2005

\section{ABSTRACT}

We present a detailed and uniform study of oxygen abundances in 155 solar type stars, 96 of which are planet hosts and 59 of which form part of a volume-limited comparison sample with no known planets. $E W$ measurements were carried out for the [OI] $6300 \AA$ line and the O I triplet, and spectral synthesis was performed for several OH lines. NLTE corrections were calculated and applied to the LTE abundance results derived from the O I 7771-5 A triplet. Abundances from [O I], the O I triplet and near-UV OH were obtained in 103, 87 and 77 dwarfs, respectively. We present the first detailed and uniform comparison of these three oxygen indicators in a large sample of solar-type stars. There is good agreement between the $[\mathrm{O} / \mathrm{H}]$ ratios from forbidden and $\mathrm{OH}$ lines, while the NLTE triplet shows a systematically lower abundance. We found that discrepancies between $\mathrm{OH},[\mathrm{OI}]$ and the $\mathrm{O}$ I triplet do not exceed 0.2 dex in most cases. We have studied abundance trends in planet host and comparison sample stars, and no obvious anomalies related to the presence of planets have been detected. All three indicators show that, on average, $[\mathrm{O} / \mathrm{Fe}]$ decreases with $[\mathrm{Fe} / \mathrm{H}]$ in the metallicity range $-0.8<[\mathrm{Fe} / \mathrm{H}]<0.5$. The planet host stars present an average oxygen overabundance of $0.1-0.2$ dex with respect to the comparison sample.

Key words. stars: abundances - stars: chemically peculiar - stars: evolution - stars: planetary systems - Galaxy: solar neighbourhood

\section{Introduction}

The discoveries of more than 120 planetary-mass companions orbiting around solar-type stars have provided important opportunities to understand the formation and evolution of planetary systems. Several studies have shown that planet-harbouring stars are on average more metal-rich than dwarfs of the same

* Based on data from the FEROS spectrograph at the 2.2-m ESO/MPI telescope (observing run ID 074.C-0135), at the La Silla Observatory, ESO (Chile), and the UVES spectrograph at VLT/UT2 Kueyen telescope (observing run ID 074.C-0134), at the Paranal Observatory, ESO (Chile), and on observations made with the SARG spectrograph at 3.5-m TNG, operated by the Fundación Galileo Galilei of the INAF, and with the UES spectrograph at the 4-m William Hershel Telescope (WHT), operated by the Isaac Newton Group, both at the Spanish Observatorio del Roque de los Muchachos of the Instituto de Astrofisica de Canarias.

$\star \star$ Tables 4-9 are only available in electronic form at http://www. edpsciences.org spectral type with no known planets (Gonzalez 1997; Gonzalez et al. 2001; Laws et al. 2003; Santos et al. 2001, 2003b, 2004a, 2005; for a review see Santos et al. 2003a). Two possible explanations have been suggested to link the metalllicity excess to the presence of planets. The first has been proposed by Gonzalez (1997), who has suggested that the iron enhancement observed in stars with planets is due mainly to the accretion of large amounts of protoplanetary material onto the star. The other hypothesis by Santos et al. $(2000,2001)$ attributes the metallicity excess of planet host stars to the high metal content of the primordial cloud out of which the planetary system formed.

Detailed chemical analysis of planet-harbouring stars can provide useful information in the understanding of how the systems with giant planets have formed. Searching for chemical anomalies related to the presence of planets, in addition to the observed iron excess, is thus of high interest in discriminating between possible planetary formation hypotheses. For instance, light elements can give important evidence of pollution events 
(Israelian et al. 2001b, 2003, 2004a; Sandquist et al. 2002; Santos et al. 2002, 2004b).

Abundance trends of volatile and refractory elements are also of interest in investigating planetary system formation. If the accretion processes were mainly responsible for the metallicity excess found in planet host stars, a relative overabundance of refractory elements would be observed, since volatiles (with low condensation temperatures) are expected to be deficient in accreted materials (Smith et al. 2001). Likewise, if planet host stars had undergone significant pollution, their volatile abundances should show clear differences with respect to those of field stars. In this framework, it is very important to achieve abundance trends for as many planet host stars and as many elements as possible, and to carry out a homogeneous comparison with field stars with no known planetarymass companion.

Several studies on abundances of metals other than iron have been carried out in planet host stars, but most of them have included only a reduced number of targets with planets and their results have been compared inhomogeneously with abundance trends of field stars from other authors (Gonzalez \& Laws 2000; Gonzalez et al. 2001; Santos et al. 2000; Takeda et al. 2001; Sadakane et al. 2002; for a review see Israelian 2004). Recently, some refractories (e.g. Ca, Ti, Si, etc.) and volatiles (N, C, S and Zn) have been analysed homogeneously in a large number of planet host targets, as well as in a comparison set of stars with no known planets (Bodaghee et al. 2003; Ecuvillon et al. 2004a,b; Beirao et al. 2005; Gilli et al. 2005). Takeda \& Honda (2005) presented a study of CNO abundances in 27 planet host stars included in a large sample of $160 \mathrm{~F}, \mathrm{G}$ and $\mathrm{K}$ dwarfs and subgiants.

Oxygen is the third most abundant element in the Universe, after hydrogen and helium. By analysing elemental abundances in the atmospheres of $F$ and $G$ dwarfs stars it is possible to determine the chemical composition of the gas out of which the stars were born and to understand the chemical evolution of the Galaxy and its formation history (e.g. McWilliam 1997). Oxygen is essentially primary. It is formed by $\alpha$-processing in massive stars and released in the interstellar medium (ISM) during type II SN explosions (e.g. Arnett 1978; Tinsley 1979; Woosley \& Weaver 1995).

Several indicators have been used in the determination of oxygen abundances in disc and halo stars: the near-IR O I triplet at 7771-5 ^ (e.g. Abia \& Rebolo 1989; Tomkin et al. 1992; King \& Boesgaard 1995; Cavallo et al. 1997; Mishenina et al. 2000; Israelian et al. 2001a; Fulbright \& Johnson 2003; Takeda 2003; Bensby et al. 2004; Schukina et al. 2005), the forbidden lines of [O I] at 6300 and $6363 \AA$ (e.g. King \& Boesgaard 1995; Fulbright \& Johnson 2003; Takeda 2003; Bensby et al. 2004; Schukina et al. 2005), and the near-UV OH lines at $3100 \AA$ (e.g. Bessell et al. 1991; Nissen et al. 1994; Israelian et al. 1998; Boesgaard et al. 1999; Israelian et al. 2001a). Unfortunately, results from different indicators show discrepancies. Israelian et al. (2004b) have reported the largest conflict between the O I triplet at 7771-5 $\AA$ and the forbidden line at $6300 \AA$ with discrepancies in $[\mathrm{O} / \mathrm{H}]$ ratios of up to 1 dex. For stars with $[\mathrm{Fe} / \mathrm{H}]<-1.0$, many studies obtained disagreement in the $[\mathrm{O} / \mathrm{Fe}]$ vs. $[\mathrm{Fe} / \mathrm{H}]$ relationship (e.g. Israelian et al. 2001a;
Nissen et al. 2002). For solar-metallicity stars as well the situation is unclear (e.g. Nissen \& Edvardsson 1992; Feltzing \& Gustafsson 1998; Prochaska et al. 2000; Bensby et al. 2004).

The analyses of these lines all have their difficulties. The triplet lines are strongly affected by deviations from LTE (e.g. Shchukina 1987; Kiselman 1991), and by convective inhomogeneities (e.g. Kiselman 1993). However newer 3D calculations by Asplund et al. (2004) showed that the NLTE effects are very similar in $1 \mathrm{D}$ and $3 \mathrm{D}$. The forbidden lines are both very weak and blended by lines from other species (e.g. Lambert 1978; Bensby et al. 2004). The $\mathrm{OH}$ lines are very sensitive to surface inhomogeneities like granulation (e.g. Kiselman \& Nordlund 1995).

Our work presents a complete and uniform study of the oxygen abundances in two large samples, a set of planetharbouring stars and a volume-limited comparison sample of stars with no known planetary-mass companions, using three different indicators in order to check the consistency of the results. We carry out a detailed comparison among the abundances provided by different lines and discuss possible discrepancies. We investigate eventual anomalies related to the presence of planets and locate our results within the framework of Galactic chemical evolution.

\section{Observations}

Most of the spectra for the [OI] line and OI triplet analysis were collected during several observational campaigns with different spectrographs: CORALIE on the $1.2 \mathrm{~m}$ Euler Swiss telescope, FEROS on the $2.2 \mathrm{~m} \mathrm{ESO/MPI} \mathrm{telescope} \mathrm{(both} \mathrm{at}$ La Silla, Chile), UVES at the VLT/UT2 Kueyen telescope (Paranal, ESO, Chile), SARG on the $3.5 \mathrm{~m}$ TNG and UES on the $4.2 \mathrm{~m}$ WHT (both at Roque de los Muchachos, La Palma, Spain). Previous works have already used them to derive stellar parameters (Santos et al. 2004a) and abundances of different species (Bodaghee et al. 2003; Ecuvillon et al. 2004a,b; Beirao et al. 2005; Gilli et al. 2005).

New optical spectra were collected with the UVES spectrograph at the Kueyen telescope, the FEROS spectrograph at the ESO/MPI telescope and the SARG spectrograph at TNG (Roque de los Muchachos, La Palma, Spain). A detailed description of the FEROS data is available in the work of Santos et al. (2005). The new optical SARG and UVES spectra have a resolution $R \sim 57000$ and $R \sim 85000$, as well as $\mathrm{S} / \mathrm{N}$ ratios above 150 and 600 , respectively, at $\sim 6000 \AA$.

For the synthesis of the $\mathrm{OH}$ lines, we used the near-UV spectra from the UVES spectrograph at the Kueyen telescope, from which Santos et al. (2002) and Ecuvillon et al. (2004a) have derived beryllium and nitrogen abundances. We refer the reader to these papers for a detailed description of the data. New near-UV spectra obtained from the UVES spectrograph at the Kueyen telescope were used. These spectra have a resolution $R \sim 75000$ and $\mathrm{S} / \mathrm{N}$ ratios above 100 in most cases at Be region. 
The data reduction for the new SARG spectra was done using $\operatorname{IRAF}^{1}$ tools in the echelle package. Standard background correction, flatfield and extraction procedures were used. The wavelength calibration was performed using a ThAr lamp spectrum taken during the same night. The FEROS and UVES spectra were reduced using the corresponding pipeline softwares.

\section{Analysis}

Abundance ratios were derived from three different indicators: the forbidden line at $6300 \AA$, the O I 7771-5 $\AA$ triplet, and a set of five near-UV OH lines at $3100 \AA$. $E W$ measurements were carried out for the [OI] line and the triplet, while spectral synthesis was performed for the $\mathrm{OH}$ lines.

LTE abundances for all the indicators were determined according to a standard analysis with the revised version of the spectral synthesis code MOOG $^{2}$ (Sneden 1973) and a grid of Kurucz (1993) ATLAS9 atmospheres with overshooting (as well as all other papers in these series). All the atmospheric parameters, $T_{\text {eff }}, \log g,[\mathrm{Fe} / \mathrm{H}]$ and $\xi_{\mathrm{t}}$, and the corresponding uncertainties, were taken from Santos et al. (2004a, 2005). The adopted solar abundances for iron, oxygen and nickel were $\log \epsilon(\mathrm{Fe})_{\odot}=7.47 \mathrm{dex}$ (as used in Santos et al. 2004a, 2005), $\log \epsilon(\mathrm{O})_{\odot}=8.74 \mathrm{dex}$ (Nissen et al. 2002), and $\log \epsilon(\mathrm{Ni})_{\odot}=$ 6.25 dex (Anders \& Grevesse 1989), respectively.

\subsection{The $[\mathrm{OI}]$ absorption at $6300 \AA$}

A standard LTE analysis was carried out to derive oxygen abundances from the [OI] absorption at $6300.3 \AA$, since it is well known that this indicator is not significantly affected by deviations from LTE (e.g. Kiselman 2001). However, this line is considerably blended by an Ni I line at $6300.399 \AA$ (e.g. Lambert 1978; Allende Prieto et al. 2001). We estimated the $E W$ of the Ni I line, using the ewfind driver of MOOG (Sneden 1973) and $\mathrm{Ni}$ abundances computed by Jorge et al. (private communication, see Fig. 11). The oxygen contribution has been obtained by subtracting the Ni $E W$ from the whole measured $E W$ of the $6300.3 \AA$ feature. The wavelengths, excitation energies of the lower levels and oscillator strengths of the Ni I absorption were taken from Allende Prieto et al. 2001), while the adopted atomic data fro [OI] are from Lambert (1978). The $\log g f$ value of the [OI] line was slightly modified in order to obtain $\log \epsilon(\mathrm{O})_{\odot}=8.74$. All these values are listed in Table 1 . Equivalent widths were determined by Gaussian fitting using the splot task of IRAF, and abundances were computed with the abfind driver of MOOG (Sneden 1973).

Uncertainties in the atmospheric parameters are of the order of $50 \mathrm{~K}$ in $T_{\text {eff }}, 0.12 \mathrm{dex}$ in $\log g, 0.08 \mathrm{~km} \mathrm{~s}^{-1}$ in the microturbulence and 0.05 dex in the metallicity (see Santos et al. 2004a, 2005). The sensitivity of the [OI] line to variations in atmospheric parameters has been estimated as follows. We selected

\footnotetext{
${ }^{1}$ IRAF is distributed by the National Optical Astronomy Observatories, operated by the Association of Universities for Research in Astronomy, Inc., under cooperative agrement with the National Science Foundation, USA.

2 The source code of MOOG2002 can be downloaded from http://verdi.as.utexas.edu/moog.html
}

Table 1. Atomic parameters adopted for [OI] $6300 \AA$ line, O I 7771-5 Å triplet and the near-UV OH lines.

\begin{tabular}{cccr}
\hline \hline Species & $\lambda(\AA)$ & $\chi_{l}(\mathrm{eV})$ & $\log g f$ \\
\hline$[\mathrm{OI}]$ & 6300.230 & 0.00 & -9.689 \\
$\mathrm{Ni} \mathrm{I}$ & 6300.399 & 4.27 & -2.310 \\
$\mathrm{O} \mathrm{I}$ & 7771.960 & 9.11 & 0.452 \\
$\mathrm{O} \mathrm{I}$ & 7774.180 & 9.11 & 0.314 \\
$\mathrm{O} \mathrm{I}$ & 7775.400 & 9.11 & 0.099 \\
$\mathrm{OH}$ & 3167.169 & 1.11 & -1.623 \\
$\mathrm{OH}$ & 3189.312 & 1.03 & -1.990 \\
$\mathrm{OH}$ & 3255.490 & 1.30 & -1.940 \\
$\mathrm{OH}$ & 3172.997 & 1.20 & -1.692 \\
$\mathrm{OH}$ & 3173.200 & 1.83 & -1.100 \\
\hline
\end{tabular}

a set of three stars having different temperatures (HD 22049, HD 37124 and HD 9826), and we then tested abundance sensitivity to changes in each atmospheric parameter $( \pm 100 \mathrm{~K}$ for $T_{\text {eff }}, \pm 0.3 \mathrm{dex}$ for $\log g$ and $[\mathrm{Fe} / \mathrm{H}], \pm 0.05 \mathrm{dex}$ for $\xi_{\mathrm{t}}$ ). The results are shown in Table 2. To take into account the uncertainties caused by the continuum determination, $E W \mathrm{~s}$ for the highest and the lowest continuum level were measured, and the corresponding abundance errors were added quadratically to the abundance uncertainties derived from the sensitivity to changes in the atmospheric parameters.

\subsection{The OI triplet at $7771-5 \AA$}

LTE abundances were derived from $E W$ measurements of the triplet lines. The wavelengths and excitation energies of the lower levels for the three triplet lines listed in Table 1 were taken from Kurucz \& Bell (1995), while oscillator strenght values consistent with $\log \epsilon(\mathrm{O})_{\odot}=8.74$ were obtained following an inverted solar analysis in LTE. Equivalent widths were determined by Gaussian fitting using the splot task of IRAF, and abundances were computed with the abfind driver of MOOG (Sneden 1973).

NLTE corrections were calculated and applied to the LTE results. The NTE computations for the oxygen atom were carried out using the atomic model with 23 levels of OI and one level of O II. Our atomic model is based on the data of Carlsson \& Judge (1993). In our computations only 31 bound-bound and 23 bound-free radiative transitions were considered; nevertheless, the consideration of additional levels and transitions does not affect our results (Shchukina 1987; Takeda 2003). It is well known that inelastic collisions with hydrogen atoms tend to offset the NLTE effects. However, it is often stated that Drawin's formalism (Drawin 1968) gives very uncertain results for hydrogen collision rates (e.g. Belyaev et al. 1999). Thus, collisions with $\mathrm{H}$ atoms were not taken into account in our computations. Figure 1 shows the dependence of the NLTE corrections on $T_{\text {eff }}, \log g$ and $[\mathrm{Fe} / \mathrm{H}]$. Our results are similar to those reported recently by Takeda (2003).

The sensitivity of the oxygen abundances derived from triplet lines to variations in atmospheric parameters has been 
Table 2. Sensitivity of the three indicators [O I], O I and OH to changes of $100 \mathrm{~K}$ in effective temperature, 0.3 dex in gravity and metallicity, and $0.5 \mathrm{~km} \mathrm{~s}^{-1}$ in microturbulence.

\begin{tabular}{cccccc}
\hline \hline & & $\Delta T_{\text {eff }}= \pm 100 \mathrm{~K}$ & $\Delta \log g= \pm 0.3$ dex & $\Delta[\mathrm{Fe} / \mathrm{H}]= \pm 0.3$ dex & $\Delta \xi_{\mathrm{t}}= \pm 0.5 \mathrm{~km} \mathrm{~s}^{-1}$ \\
\hline$[\mathrm{O} \mathrm{I}]$ & Star & $\Delta[\mathrm{O} / \mathrm{H}]$ & $\Delta[\mathrm{O} / \mathrm{H}]$ & $\Delta[\mathrm{O} / \mathrm{H}]$ & $\Delta[\mathrm{O} / \mathrm{H}]$ \\
\hline & HD 22049 & \pm 0.01 & \pm 0.14 & \pm 0.09 & $\pm 0.00^{1}$ \\
& HD 37124 & \pm 0.01 & \pm 0.14 & \pm 0.08 & $\pm 0.00^{1}$ \\
& HD 9826 & \pm 0.01 & \pm 0.14 & \pm 0.09 & \pm 0.01 \\
\hline O I & Star & $\Delta[\mathrm{O} / \mathrm{H}]$ & $\Delta[\mathrm{O} / \mathrm{H}]$ & $\Delta[\mathrm{O} / \mathrm{H}]$ & $\Delta[\mathrm{O} / \mathrm{H}]$ \\
\hline & HD 22049 & $\mp 0.14$ & \pm 0.09 & \pm 0.02 & $\mp 0.02$ \\
& HD 37124 & $\mp 0.10$ & \pm 0.05 & \pm 0.02 & $\mp 0.02$ \\
& HD 9826 & $\mp 0.07$ & \pm 0.05 & \pm 0.02 & $\mp 0.03$ \\
\hline OH & Star & $\Delta[\mathrm{O} / \mathrm{H}]$ & $\Delta[\mathrm{O} / \mathrm{H}]$ & $\Delta[\mathrm{O} / \mathrm{H}]$ & $\Delta[\mathrm{O} / \mathrm{H}]$ \\
\hline & HD 22049 & \pm 0.05 & $\mp 0.03$ & \pm 0.18 & $\pm 0.00^{1}$ \\
& HD 37124 & \pm 0.10 & $\mp 0.05$ & \pm 0.20 & $\pm 0.00^{1}$ \\
& HD 9826 & \pm 0.12 & $\mp 0.05$ & \pm 0.20 & $\pm 0.00^{1}$ \\
\hline
\end{tabular}

${ }^{1}$ These sensitivities are based on the method described in Sect. 3.1. The values can be slightly larger if more explicit calculations are carried out.

estimated in the same way as in the [OI] case (see Sect. 3.1). The results are shown in Table 2. Uncertainties in the final oxygen abundances were determined adding in quadrature the abundance uncertainty resulting from the continuum determination $(0.05 \mathrm{dex})$, the standard deviation of each mean abundance and the errors due to the abundance sensivities to changes in the atmospheric parameters.

\subsection{Synthesis of the near-UV OH lines}

We determined oxygen abundances by fitting synthetic spectra to the data. Four $\mathrm{OH}$ features were analysed at $3167.2 \AA$, at $3189.3 \AA$, at $3255.5 \AA$, and at $3173 \AA$, composed of two $\mathrm{OH}$ lines, at $3172.9 \AA$ and at $3173.2 \AA$. The atomic line lists for each spectral region were taken from VALD (Kupka et al. 1999), while the molecular data of the OH lines were extracted from Kurucz database ${ }^{3}$. We assumed the dissociation potential of the $\mathrm{OH}$ molecule $D_{0}(\mathrm{OH})=4.39 \mathrm{eV}$ (Huber $\&$ Herzberg 1979). Oscillator strength values were slightly modified in order to achieve a good fit to the Kurucz Solar Atlas (Kurucz et al. 1984), with a solar model having $T_{\text {eff }}=5777 \mathrm{~K}$, $\log g=4.44 \mathrm{dex}$, and $\xi_{\mathrm{t}}=1.0 \mathrm{~km} \mathrm{~s}^{-1}$. The adopted data are listed in Table 1.

The continuum was normalized with 5 th order polynomials using the CONT task of IRAF. We then made further improvements in the placement of the continuum using the DIPSO task of the STARLINK software: some points of reference of the continuum level were selected in the Kurucz Solar Flux Atlas (Kurucz et al. 1984) and used in the determination of the stellar continuum of our observed spectra. For the instrumental broadening we used a Gaussian function with FWHM of $0.05 \AA$ and a rotational broadening function with $v \sin i$ values from CORALIE database. All our targets are slow rotators, with $v \sin i$ values between 1 and $5 \mathrm{~km} \mathrm{~s}^{-1}$ in almost all cases.

\footnotetext{
${ }^{3}$ Molecular line data can be downloaded at http://kurucz.harvard.edu/LINELISTS/LINESMOL
}

No macroturbolence broadening was used. Two examples of the fitting of the four features are shown in Fig. 2.

The sensitivity of the oxygen abundances from $\mathrm{OH}$ lines to changes in the atmospheric parameters was estimated in the same way as for the [OI] and OI indicators (see Sects. 3.1 and 3.2). Uncertainties derived from inaccuracies in atmospheric parameters were added in quadrature to the abundance uncertainty resulting from the continuum determination $(0.05 \mathrm{dex})$ and to the standard deviation of each mean abundance.

The dependence on $T_{\text {eff }}$ and on $\log g$ of the $[\mathrm{O} / \mathrm{H}]$ results from all the indicators is represented in Fig. 3. We note that no significant trends appear for [O I] and $\mathrm{OH}$. This means that our results are almost free from systematic errors. Only in the case of triplet, does a trend of decreasing $[\mathrm{O} / \mathrm{H}]$ with increasing $T_{\text {eff }}$ exist. This is probably due to the high dependence on $T_{\text {eff }}$ of the NLTE corrections applied to the LTE results of the triplet.

\section{Comparison between different indicators}

Abundances from the [OI] line at $6300 \AA$ were obtained in 103 dwarfs (see Tables 4 and 5), while 77 stars were analysed using near-UV OH lines (see Tables 6 and 7). LTE abundances were derived from the triplet lines at 7771-5 $\mathrm{A}$ and then corrected for NLTE effects for 87 stars (see Tables 8 and 9). Altogether we present oxygen abundances derived from these three indicators for 96 and 59 stars with and without known planets, respectively.

\subsection{Triplet vs. [OI] and near-UV OH}

Figure 4 (top panel) shows the comparison between the results obtained from the NLTE study of the 7771-5 $\AA$ triplet and the analysis of the [OI] $6300 \AA$ line. NLTE triplet abundances are systematically lower with respect to the forbidden-line results, with discrepancies of the order of 0.3 dex on average. 

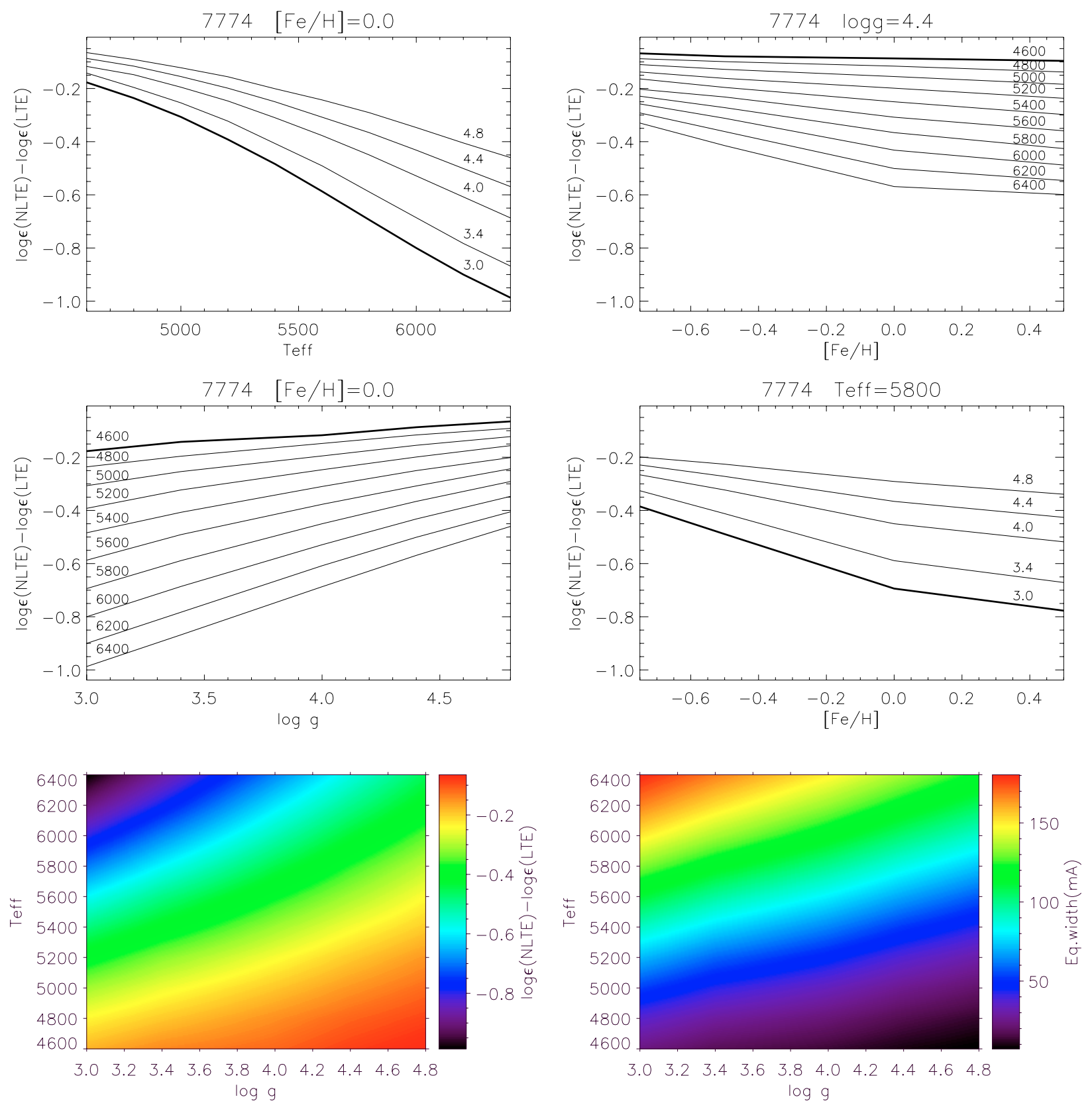

Fig. 1. NLTE abundance corrections $\log \epsilon(N L T E)-\log \epsilon(L T E)$ and NLTE equivalent width of the OI IR triplet $\lambda 7774 \AA$ line in a grid of one-dimensional model atmospheres of Kurucz spanning the range $4600 \mathrm{~K}<T_{\text {eff }}<6400 \mathrm{~K}, 3.0<\log g<4.8$, and $-0.75<[\mathrm{Fe} / \mathrm{H}]<0.5$. The right-hand top and the right-hand middle panels show the results for stars with the solar-like gravity $\log g=4.4$ and the solar-like effective temperature $T_{\text {eff }}=5800 \mathrm{~K}$, while the right-hand bottom and all the left-hand panels show the results for stars with the solar metallicity $[\mathrm{Fe} / \mathrm{H}]=0.0$. The bottom panels show variations of the NLTE abundance corrections (left) and the NLTE equivalent width with $T_{\mathrm{eff}}$ and $\log g$ for $[\mathrm{Fe} / \mathrm{H}]=0$. The numbers above curves indicate the gravities and effective temperatures.

The values obtained from the synthesis of $\mathrm{OH}$ lines present a better consistency with the NLTE triplet results (see Fig. 4, middle panel). In this case, the comparison is less meaningful because of the limited number of targets in common between the two analyses. Nevertheless, a large portion of targets show underabundances of the order of 0.2 dex in NLTE triplet values with respect to near-UV OH results.

The NLTE corrections applied to the LTE analysis results correspond to the maximum effect, since collisions with $\mathrm{H}$ atoms are not taken into account (see Sect. 3.2). This can produce an underestimation of the final triplet abundances, and could be the reason for the systematic underabundance of the NLTE triplet results.

If we compare results from LTE triplet analysis with those from [OI] $6300 \AA$ and OH (see Fig. 5), the consistency with these indicators improves, with typical discrepancies of 0.1 dex. Moreover, a suggestive number of targets shows an overabundance of the order of $0.2 \mathrm{dex}$ in LTE triplet values. This means that the NLTE and LTE analyses give lower and upper limits, respectively, for the oxygen abundance. 

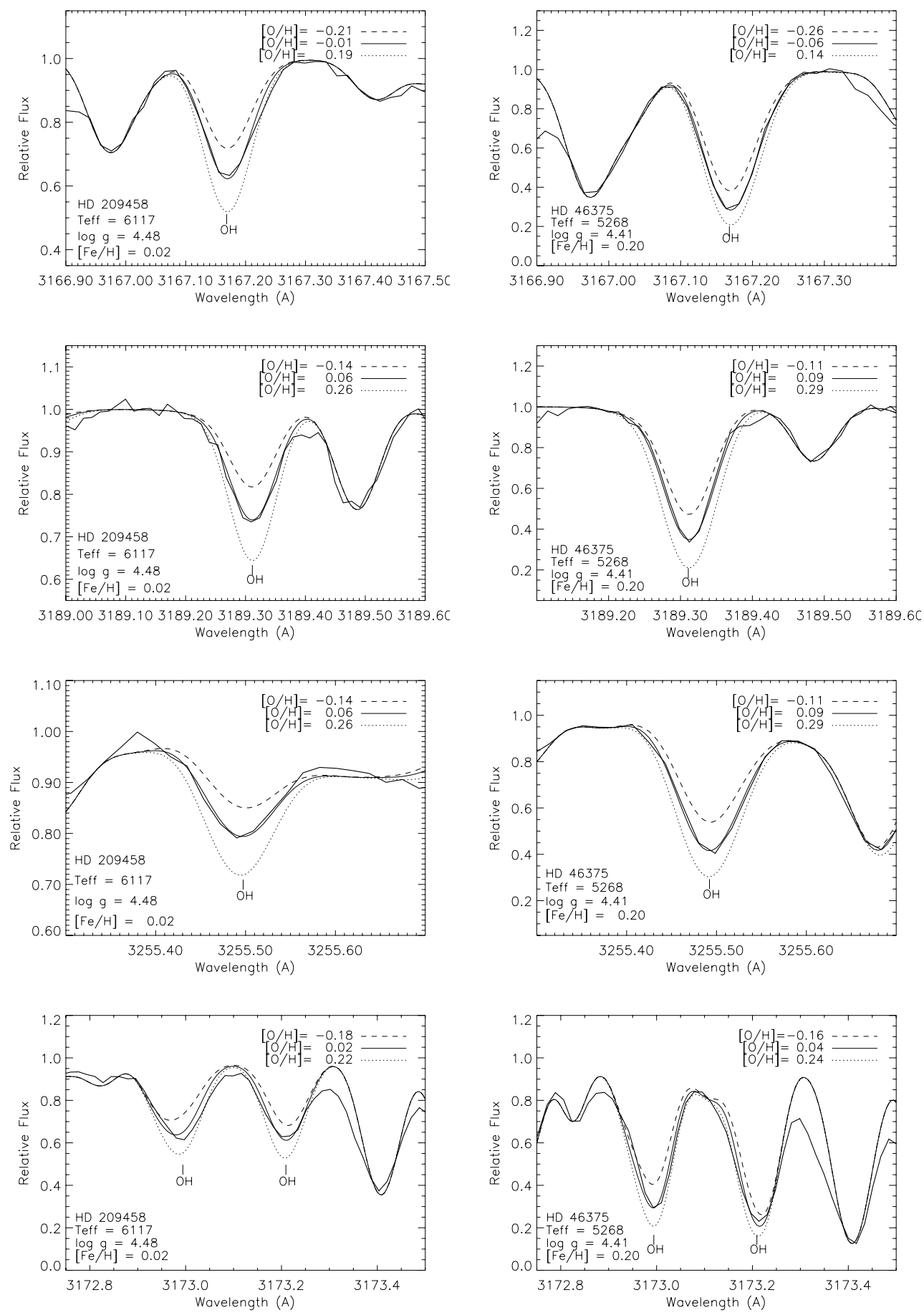

Fig. 2. The observed spectrum (thick solid line) and three synthetic spectra (dotted, dashed and solid lines) for different values of [O/Fe] in the four $\mathrm{OH}$ features analysed, for two targets.

\section{2. [OI] vs. near-UV OH}

Figure 4 (bottom panel) represents the comparison between oxygen abundances obtained from the analysis of the [O I] $6300 \AA$ line and from the synthesis of near-UV OH lines. In most cases the two indicators agree quite well, with discrepancies of the order of 0.1 dex. Abundances obtained from $\mathrm{OH}$ lines are generally lower than those from the $6300 \AA$ [O I] line.
The cause of this behaviour could be an underestimation of the Ni I component blended with the $6300 \AA$ [O I] line. The atomic parameters, especially the $\log g f$, for this $\mathrm{Ni}$ I line are uncertain (e.g. Allende Prieto et al. 2001), and this can introduce uncertainities in the estimation of the Ni I line contribution to the total spectral feature at $6300.3 \AA$. Moreover, dwarfs have very weak $[\mathrm{OI}]$ lines (e.g. $E W \sim 5 \mathrm{~m} \AA$ in the Sun). Thus, measurement uncertainties may be very large, depending on the resolution and $\mathrm{S} / \mathrm{N}$ of the data. 

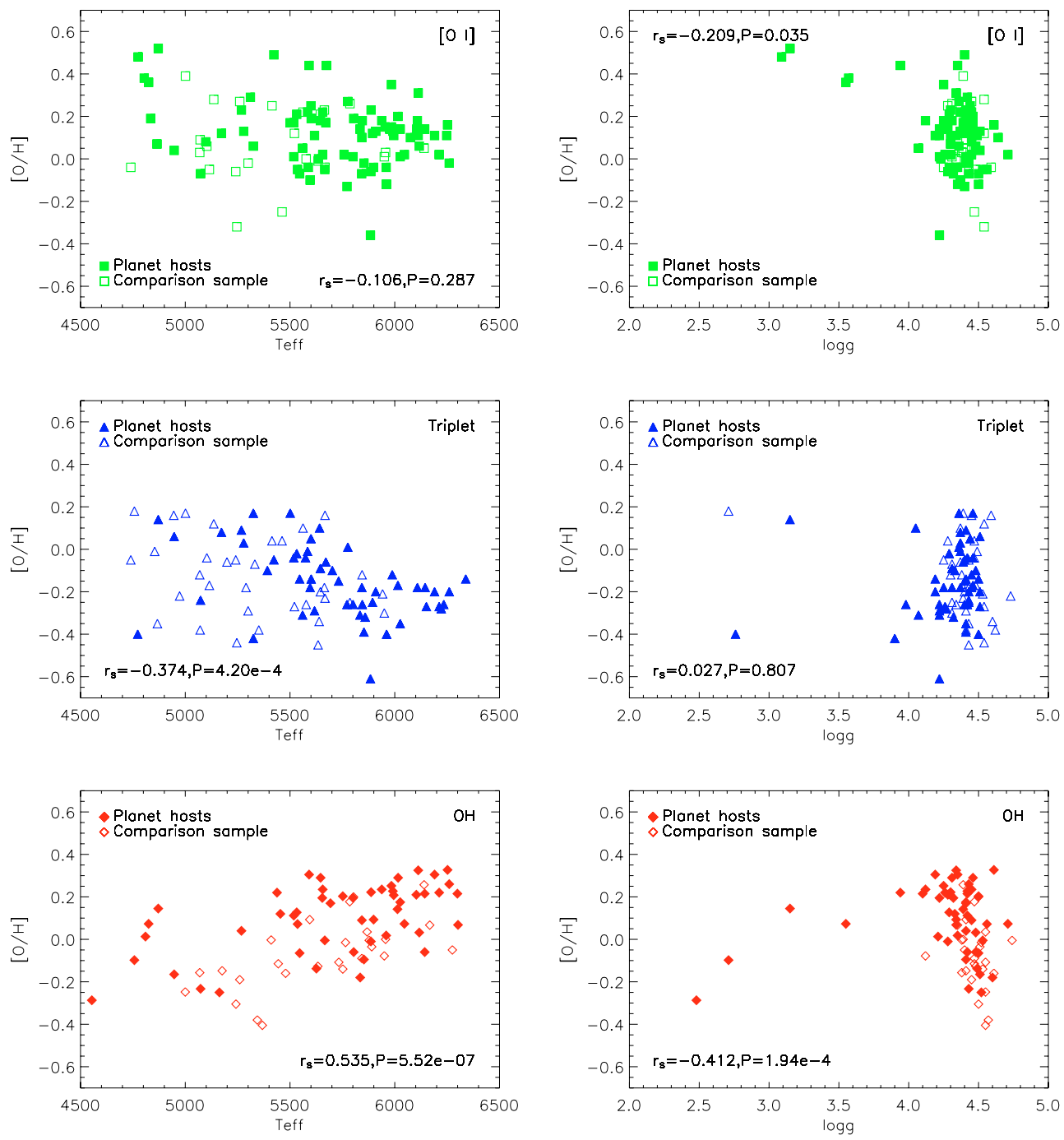

Fig. 3. $[\mathrm{O} / \mathrm{Fe}]$ vs. $T_{\text {eff }}$ and $\log g$ for the different indicators. Filled and open symbols represent planet host and comparison sample stars, respectively. The Spearman rank-order correlation coefficient and its significance value are written at the bottom of each plot.

If the uncertainties related to Ni I were the main responsible of the discrepancies with the other two indicators, a correlation should exist between these discrepancies and metallicity. Figure 6 shows the difference of abundances from $[\mathrm{OI}]$ and from other indicators as a function of $[\mathrm{Fe} / \mathrm{H}]$, as well as the correlation coefficient and its significance value. Since no correlation with metallicity exists, we can discard the possibility that the Ni I blend introduces significant errors into our [O I] results.

Another source of uncertainty could be the strong dependence of the $\mathrm{OH}$ lines on temperature, and therefore to surface inhomogeneities. However, previous works (e.g. Israelian et al. 1998; Boesgaard et al. 1999; Ecuvillon et al. 2004a) obtained a good consistence between abundances based on molecular and atomic features using classical 1D atmosphere models. This fact, added to the agreement we have found between [OI] and $\mathrm{OH}$ measures, makes us confident about the reliability of our $\mathrm{OH}$ results. We found that discrepancies between $\mathrm{OH}, \mathrm{O}$ I triplet and [O I] barely exceed $0.2 \mathrm{dex}$.

\section{Comparison between planet host and comparison sample stars}

Several studies have been published about abundances of metals other than iron in planet host stars (Santos et al. 2000; Gonzalez et al. 2001; Takeda et al. 2001; Sadakane et al. 2002; Bodaghee et al. 2003; Ecuvillon et al. 2004a,b). Oxygen abundances have been analysed by some of them (Santos et al. 2000; Gonzalez et al. 2001; Takeda et al. 2001; Sadakane et al. 2002; Takeda \& Honda 2005). However the limited number of planet host stars considered, and the comparison realized by some authors between targets with planets and field stars extracted from the literature, have prevented definitive conclusions from being reached.

We have carried out a homogeneous study of oxygen abundances in an almost complete set of 96 stars with extrasolar giant planets, as well as in a large volume-limited sample of 59 stars with no known planetary-mass companion, all belonging to the CORALIE planet search survey (see 

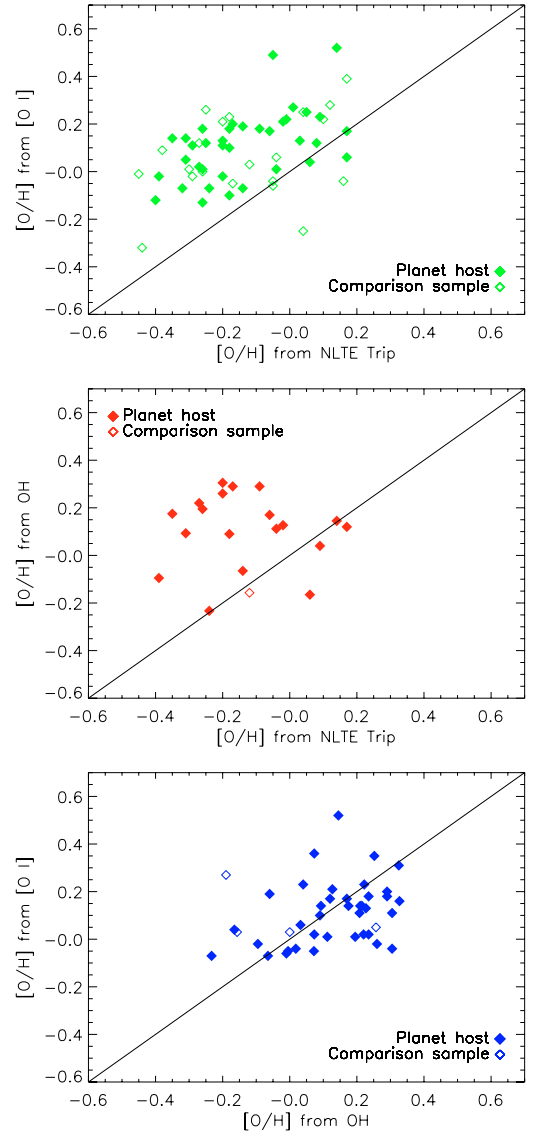

Fig. 4. Comparisons of the results from different indicators: $[\mathrm{O}]$ line, $\mathrm{OH}$ lines and triplet lines in NLTE.
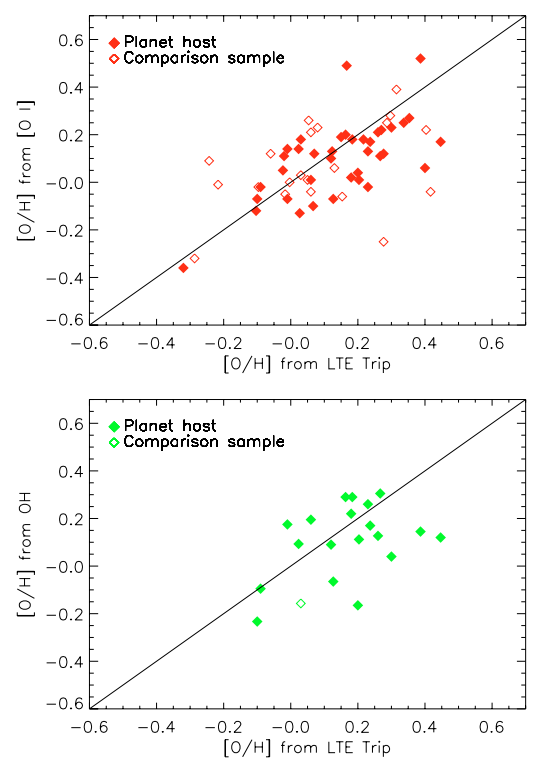

Fig. 5. Comparisons of the results from $[\mathrm{O} \mathrm{I}]$ and $\mathrm{OH}$ lines with those obtained from triplet lines in LTE.

Udry et al. 2000). The volume-limited comparison sample consists of stars from the CORALIE southern planet search sample without any known planets, with distances below $20 \mathrm{pc}$, as derived from Hipparcos parallaxes (ESA 1997). All these stars

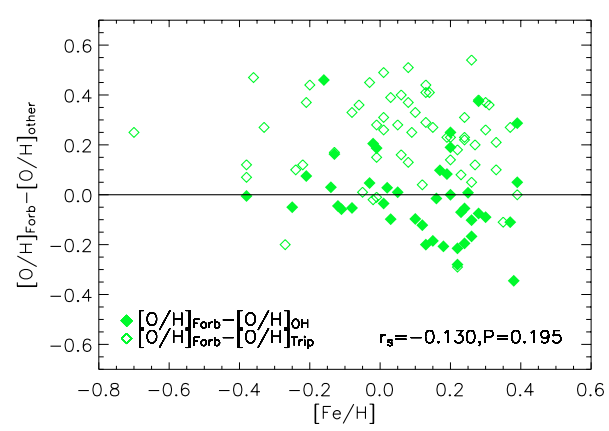

Fig. 6. Difference between $[\mathrm{O} / \mathrm{H}]$ ratios derived from $[\mathrm{OI}]$ and from another indicator, $\mathrm{OH}$ (filled symbols) or triplet (open symbols), vs. $[\mathrm{Fe} / \mathrm{H}]$. The Spearman rank-order correlation coefficient and its significance value are written at the bottom of the plot.

and their parameters come from Santos et al. (2001, 2003b, 2004a, 2005). Three different indicators were used in order to obtain more reliable and solid results.

\section{1. $[\mathrm{O} / \mathrm{H}]$ distributions}

Figure 7 presents $[\mathrm{O} / \mathrm{H}]$ distributions of the two samples, stars with and without planets, for the different indicators: [OI] $6300 \AA$ (left top panel), near-UV OH (top right panel) and triplet 7771-5 $\AA$ with NLTE (bottom left panel) and LTE (bottom right panel) treatments. Forbidden line results for the comparison sample present a bimodal shape, characterized by a steep descent from the peak around $[\mathrm{O} / \mathrm{H}] \sim 0.0$ towards negative values. The distribution of planet host stars obtained with the same indicator exhibits a symmetrical shape, with a steep descent from the peak around $[\mathrm{O} / \mathrm{H}] \sim 0.2$ towards $[\mathrm{O} / \mathrm{H}]>0.2$. An asymmetric distribution is obtained from the $\mathrm{OH}$ results for planet host stars, while the comparison sample has a symmetric shape (see Fig. 7, top right panel). The NLTE and LTE triplet distributions are quite symmetrical for the two samples of stars with and without planets (see Fig. 7, bottom panels).

The average values of $[\mathrm{O} / \mathrm{H}]$ for the samples with and without planets for each indicator, and for all the indicators together, as well as the rms dispersions and the differences between the mean $[\mathrm{O} / \mathrm{H}]$ values, are listed in Table 3 . In general, the mean $[\mathrm{O} / \mathrm{H}]$ value corresponding to the comparison sample is lower than the mean abundance value obtained for the set of planet host stars. From the [OI] analysis we obtain a difference of the order of 0.05 dex between the mean abundances of the two samples, while the $\mathrm{OH}$ synthesis leads to a larger difference of 0.17 dex.

The NLTE triplet values present a particular characteristic: the mean abundance value is lower in planet host stars than in the comparison sample. This could be because the comparison stars with available triplet measurements are generally cooler than planet host stars with triplet determinations, and NLTE corrections are much less important for lower $T_{\text {eff }}$. The abundance underestimation related to our NLTE treatment (see Sect. 4.1) is therefore on average less significant in the comparison sample than in the planet host stars. In fact, if we select two subsamples of stars with and without planets having the same $T_{\text {eff }}$ distributions, and compare their triplet $[\mathrm{O} / \mathrm{H}]$ 

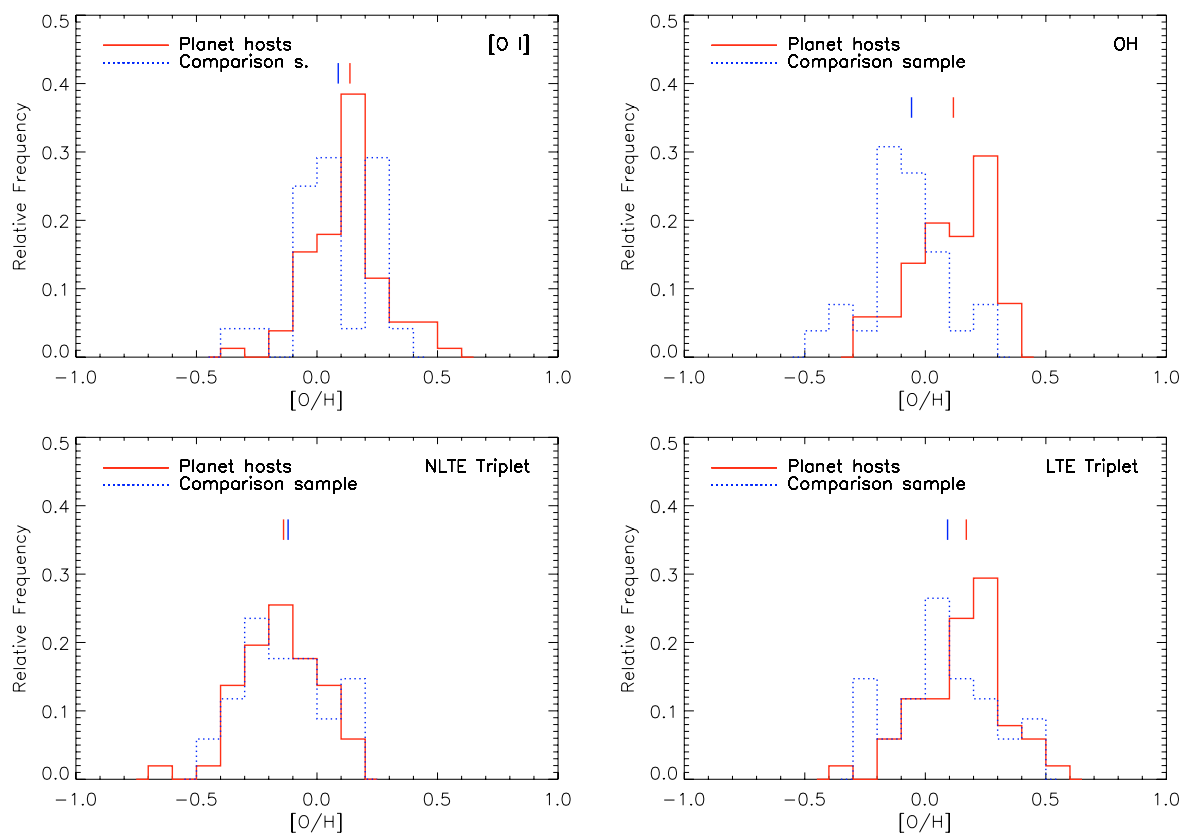

Fig. 7. $[\mathrm{O} / \mathrm{H}]$ distributions from different indicators. The solid and dotted lines represent planet host and comparison sample stars, respectively. The vertical lines represent the average abundance values of the two samples, stars with and without planets.

Table 3. Average $[\mathrm{O} / \mathrm{H}]$ values from the different indicators, with the corresponding dispersions, for the set of planet host stars and the comparison sample.

\begin{tabular}{cccr}
\hline \hline Indicator & $\begin{array}{c}\langle[\mathrm{O} / \mathrm{H}]\rangle \pm \text { rms } \\
\text { (comp. sample) }\end{array}$ & $\begin{array}{c}\langle[\mathrm{O} / \mathrm{H}]\rangle \pm \text { rms } \\
\text { (planet hosts) }\end{array}$ & Difference \\
\hline OH & $-0.07 \pm 0.16$ & $0.10 \pm 0.16$ & 0.17 \\
[O I] & $0.07 \pm 0.17$ & $0.12 \pm 0.16$ & 0.05 \\
NLTE O I & $-0.14 \pm 0.18$ & $-0.16 \pm 0.17$ & -0.02 \\
LTE O I & $0.08 \pm 0.20$ & $0.15 \pm 0.17$ & 0.07 \\
mean (NLTE) & $-0.07 \pm 0.16$ & $0.03 \pm 0.18$ & 0.10 \\
mean (LTE) & $-0.07 \pm 0.16$ & $0.12 \pm 0.16$ & 0.19 \\
\hline
\end{tabular}

distributions, this effect disappears (see Fig. 8). The planet host subsample shows an average oxygen overabundance of the order of 0.1 dex with respect to the comparison subsample, in both NLTE (Fig. 8, left panel) and LTE (Fig. 8, right panel) analyses.

Figure 9 presents the $[\mathrm{O} / \mathrm{H}]$ distributions of the two samples, stars with and without planets, obtained by averaging for each target the abundances obtained from different indicators, adopting NLTE (left panel) or LTE (right panel) triplet values. The distributions of the comparison sample obtained in both cases are very similar: both have slightly asymmetric shapes, with mean values of -0.10 dex. Concerning the planet host sample, both distributions issued from including NLTE and LTE triplet values, respectively, show asymmetric shapes. The latter present a mean value $(\langle[\mathrm{O} / \mathrm{H}]\rangle=0.12)$ much larger than the former $(\langle[\mathrm{O} / \mathrm{H}]\rangle=0.03-$ see Table 3$)$. As our NLTE treatement considers maximum NLTE corrections, we may consider the abundances obtained from including the NLTE and LTE triplet values to correspond to lower and upper limits, respectively (see Sect. 4.1). Therefore we propose that planet host stars present an average oxygen overabundance between 0.1 and 0.2 dex with respect to the comparison sample.

\section{2. $[\mathrm{O} / \mathrm{H}]$ and $[\mathrm{O} / \mathrm{Fe}]$ vs. $[\mathrm{Fe} / \mathrm{H}]$}

In Fig. 10, $[\mathrm{O} / \mathrm{Fe}]$ and $[\mathrm{O} / \mathrm{H}]$ ratios as functions of $[\mathrm{Fe} / \mathrm{H}]$ for [OI] $6300 \AA$ (top panel), O I 7771-5 $\AA$ (middle panel), and near-UV OH (bottom panel) are presented. No clear differences appear between the behaviours of the two samples, stars with and without planets. There seem to be no anomalies in oxygen abundances related to the presence of planets. The average trends that planet host stars mark are similar to those traced by the comparison sample, although discrepancies between the two trends are slightly larger for the triplet than for the other indicators. Since targets with planets are on average more metal rich than comparison sample stars, their abundance distributions correspond to the extensions of the comparison sample trends at high $[\mathrm{Fe} / \mathrm{H}]$.

The average trends resulting from different indicators present slight discrepancies, but similar behaviours. The abundances obtained from $\mathrm{OH}$ line synthesis present less dispersion than those derived from the other two indicators. The $[\mathrm{O} / \mathrm{Fe}]$ vs. $[\mathrm{Fe} / \mathrm{H}]$ plots for all the indicators show that, on average, $[\mathrm{O} / \mathrm{Fe}]$ clearly decreases with $[\mathrm{Fe} / \mathrm{H}]$ in the metallicity range $-0.8<[\mathrm{Fe} / \mathrm{H}]<0.5$, with significantly negative slopes in all the linear least-squares fits. The linear least-squares fit for the $[\mathrm{O} / \mathrm{Fe}]$ values averaged from the three indicators gives a slope of $-0.50 \pm 0.04$.

Bensby et al. (2004) obtained a similar trend of decreasing $[\mathrm{O} / \mathrm{Fe}]$ with increasing $[\mathrm{Fe} / \mathrm{H}]$ by analyzing $[\mathrm{OI}]$ in a large set of disk dwarfs. This behaviour could be caused by the steep rise these authors found in $[\mathrm{Ni} / \mathrm{Fe}]$ vs. $[\mathrm{Fe} / \mathrm{H}]$ for $[\mathrm{Fe} / \mathrm{H}]>0$. Nevertheless, since our $[\mathrm{Ni} / \mathrm{Fe}]$ vs. $[\mathrm{Fe} / \mathrm{H}]$ plot does not show 

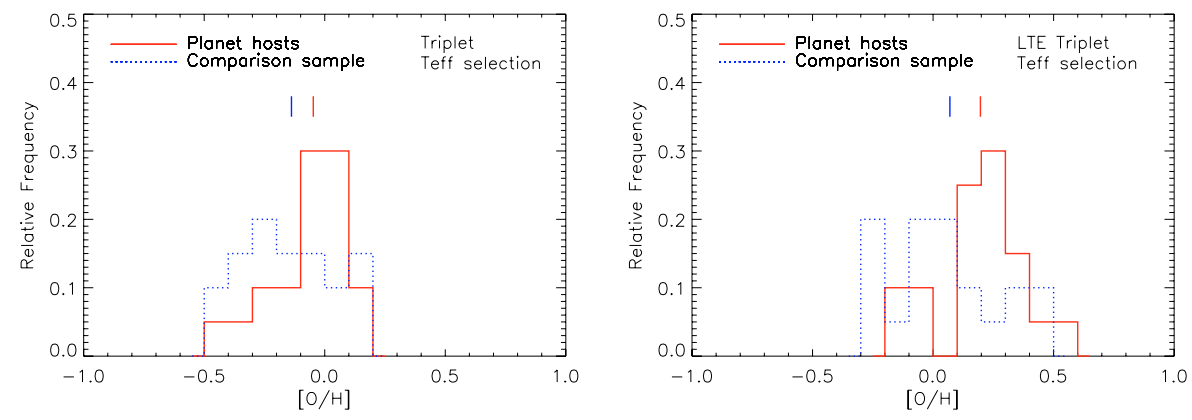

Fig. 8. $[\mathrm{O} / \mathrm{H}]$ distributions for NLTE (left panel) and LTE (right panel) triplet results for the two subsamples of planet host (solid line) and comparison sample (dotted line) stars with the same $T_{\text {eff }}$ distributions. The vertical lines represent the average abundance values of the two subsamples, stars with and without planets.
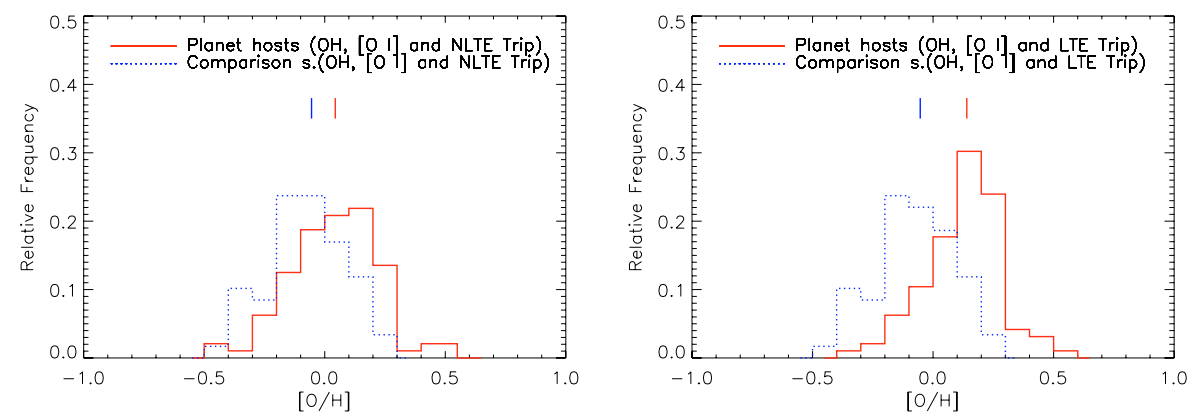

Fig. 9. Left panel: $[\mathrm{O} / \mathrm{H}]$ distributions using average results from $\mathrm{OH}$ lines, $[\mathrm{OI}]$ line and $\mathrm{O}$ I triplet in NLTE. Right panel: $[\mathrm{O} / \mathrm{H}]$ distributions using average results from $\mathrm{OH}$ lines, [O I] line and O I triplet in LTE. The solid and dotted lines represent planet host and comparison sample stars, respectively. The vertical lines represent the average abundance values of the two samples, stars with and without planets.

such a obvious increase (see Fig. 11), it is very unlikely that our [OI] results are affected by this phenomenon. Moreover, the three indicators reproduce similar steep descents, which additionally supports that the $[\mathrm{O} / \mathrm{Fe}]$ decrease with increasing $[\mathrm{Fe} / \mathrm{H}]$ found from $[\mathrm{OI}]$ analysis is "real".

\section{Galactic trends or effects related with planets?}

The abundances of volatile elements are a key factor in searching for chemical anomalies associated with the presence of planets. If the accretion of large amounts of planetary material were the dominant source of the metallicity excess observed in planet host stars, a relative overabundance of refractory elements with respect to volatiles, or at least some anomaly related to the presence of planets, would be expected in the majority of these kinds of targets (e.g. Smith et al. 2001). Thus knowing how the abundances of volatile and refractory elements behave in stars with and without planets can give valuable clues to the relative importance of the differential accretion.

Our results show that the oxygen abundances do not present clear anomalies in planet host stars with respect to comparison sample dwarfs. The trends traced by the two samples, stars with and without planets, are nearly indistinguishable. This supports the "primordial" hypothesis suggested by Santos et al. (2000, 2001), which proposes the high metal content of the protoplanetary cloud the system planets-star has formed out of as an explanation for the observed iron overabundance in planet-harbouring stars. Likewise, although the occurrence of accretion is not excluded, the possibility that pollution is the principal source of the observed metallicity enhancement is unlikely. Therefore the observed trends would simply be a product of the chemical evolution of the Galaxy.

Previous studies have already led to results supporting a "primordial" origin of the iron excess in planet host stars (Pinsonneault et al. 2001; Santos et al. 2001, 2003b, 2004a, 2005). Takeda et al. (2001) and Sadakane et al. (2002) found no differences between the abundances of some refractory and volatile elements for a set of planet host stars and some field dwarfs from the literature. Recently, Ecuvillon et al. (2004a,b) have found that the volatiles $\mathrm{N}, \mathrm{C}, \mathrm{S}$, and $\mathrm{Zn}$ behave identically in a large set of planet host and comparison sample stars analysed homogeneously. Similar results have been found by Takeda \& Honda (2005) for CNO abundances in a set of 27 planet host stars.

We have also obtained a clear monotonic decrease of $[\mathrm{O} / \mathrm{Fe}]$ with $[\mathrm{Fe} / \mathrm{H}]$ in the metallicity range $-0.8<[\mathrm{Fe} / \mathrm{H}]<0.5$ for all the spectroscopic indicators. The corresponding linear least-squares fits have significantly negative slopes, with values around -0.5 . Some previous studies of oxygen abundances in Galactic disc stars (Nissen \& Edvardsson 1992; Edvardsson et al. 1993; Nissen et al. 2002) found [O/Fe] ratios flattening in the metallicity range $-0.3<[\mathrm{Fe} / \mathrm{H}]<0.3$. Nevertheless, further studies have revealed that oxygen continues to decline with increasing [Fe/H] (e.g. Feltzing \& Gustafsson 1998; Takeda 2003). A recent analysis of a large number of $F$ and $G$ disk dwarfs by Bensby et al. (2004) has obtained a monotonic decrease of $[\mathrm{O} / \mathrm{Fe}]$ ratios in the metallicity range $-0.9<[\mathrm{Fe} / \mathrm{H}]<$ 0.4 , which is in concordance with the predictions of chemical 

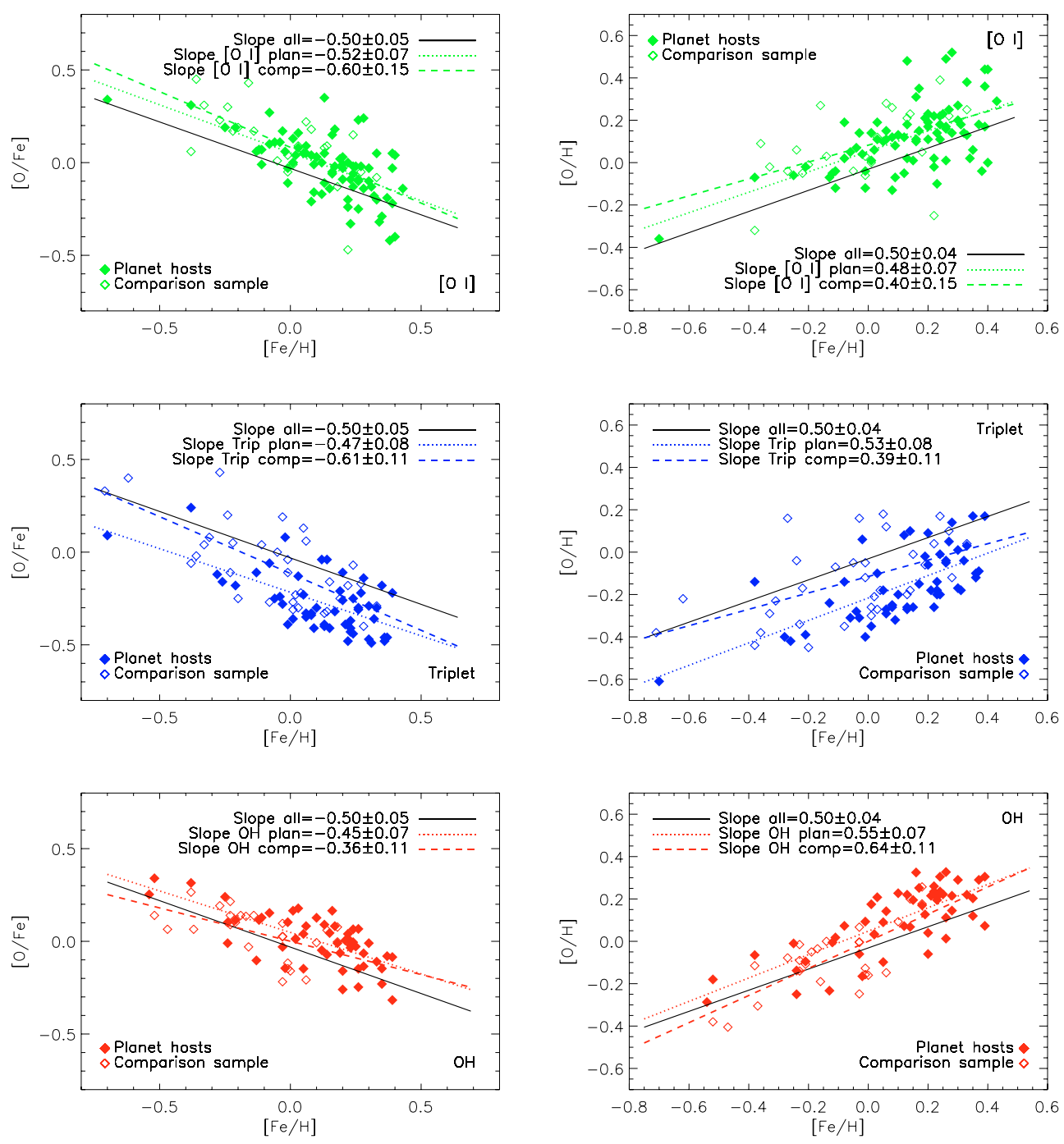

Fig. 10. $[\mathrm{O} / \mathrm{Fe}]$ and $[\mathrm{O} / \mathrm{H}]$ vs. $[\mathrm{Fe} / \mathrm{H}]$ plots for the three indicators. Filled and open symbols represent planet host and comparison sample stars, respectively. Linear least-squares fits to both samples, stars with and without planets, for each indicator and for the three indicators together are represented and slope values are indicated at the bottom of each plot.

evolution models of the Milky Way (e.g. Chiappini et al. 2003). This implies that oxygen is produced only in SNe II, with no SN Ia signature contribution, which would produce a levelling out of $[\mathrm{O} / \mathrm{Fe}]$ at $[\mathrm{Fe} / \mathrm{H}]=0$, as has been observed in other $\alpha$ elements (Bensby et al. 2003, 2004). Takeda \& Honda (2005) have found $[\mathrm{O} / \mathrm{Fe}]$ increasing with decreasing $[\mathrm{Fe} / \mathrm{H}]$ with a slope of $\sim 0.4-0.5$ for a large sample of 160 dwarfs with metallicities $-1<[\mathrm{Fe} / \mathrm{H}]<+0.4$. Our study has obtained a similar monotonic decrease and thus confirms this issue.

An average oxygen overabundance of between 0.1 and 0.2 dex in the planet host stars with respect to the comparison sample has been obtained. It is not clear if this difference is due to the presence of planets. In order to check this possibility, models of Galactic chemical evolution in this metallicity range must be studied. Unfortunately, such models are not available, and our conclusions can only be based on the best guess. For example, assuming that the main parameters which govern the trends of chemical elements in the galactic disk do not vary

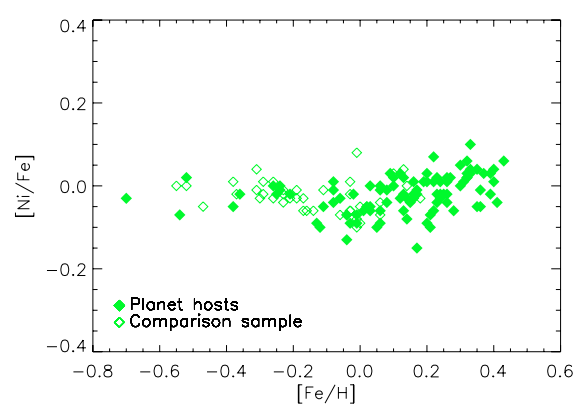

Fig. 11. $[\mathrm{Ni} / \mathrm{Fe}]$ vs. $[\mathrm{Fe} / \mathrm{H}]$ plot. Filled and open symbols represent planet host and comparison sample stars, respectively.

in the metallicity range $-1 .<[\mathrm{Fe} / \mathrm{H}]<0.5$, we would expect that the elements of the same nuclesynthetic origin present similar behaviours at super-solar metallicities. However, this is not what is observed. For instance, the $[\mathrm{X} / \mathrm{Fe}]$ ratios of the $\alpha$-elements $\mathrm{Si}, \mathrm{Ti}$ and $\mathrm{Mg}$ decrease monotonically in the 
metallicity range $-1<[\mathrm{Fe} / \mathrm{H}]<0$ and become constant in the super-solar regime (e.g. Bodaghee et al. 2003; Gilli et al. 2005), while, as we have seen above, the $[\mathrm{O} / \mathrm{Fe}]$ ratio continously decreasing in the whole metallicity range. Since at these metallicities the fraction of planet-harbouring stars is important, we cannot exclude the possibility of global effects on abundance trends linked to planets. More works needs to be done before we can tackle this problem.

\section{Concluding remarks}

We presented the first detailed and uniform study of oxygen abundances in an almost complete set of planet-harbouring stars and in a unbiased volume-limited comparison sample of solar-type dwarfs with no known planetary-mass companions. An homogeneous set of atmospheric parameters, spectroscopically determined, was adopted, and three independent analyses from different indicators were performed. We also provide the first accurate and homogeneous comparison of near-UV OH, $6300 \AA[\mathrm{OI}]$ and 7771-5 $\mathrm{O}$ O I triplet in a large set of solartype stars.

Oxygen is one of the most controversial elements due to its problematic indicators. We found a good agreement between the results of [O I] and $\mathrm{OH}$ analyses. The NLTE treatement for the triplet led to an underestimation of the oxygen abundances, while the LTE values may be considered as abundance upper limits. The $\mathrm{OH}$ results show a much smaller dispersion in the $[\mathrm{O} / \mathrm{Fe}]$ and $[\mathrm{O} / \mathrm{H}]$ vs. $[\mathrm{Fe} / \mathrm{H}]$ plots with respect to the others values, while the $[\mathrm{O} \mathrm{I}]$ analysis generally reveals a better agreement with other indicators.

We found that $[\mathrm{O} / \mathrm{Fe}]$ and $[\mathrm{O} / \mathrm{H}]$ trends as function of metallicity show the same behaviour in planet host and comparison sample stars. No anomalies associated with the presence of planets have appeared in those representations. For all the indicators, $[\mathrm{O} / \mathrm{Fe}]$ ratios decrease monotonically with $[\mathrm{Fe} / \mathrm{H}]$, with significantly negative slopes of the order of -0.5 . Planet host stars present on average an oxygen overabundance between 0.1 and 0.2 dex with respect to the comparison sample.

We have discussed whether these characteristics are effects related to the presence of planets or products of Galactic chemical evolution. Further investigations on the refractory/volatile abundance ratios in stars with and without planets, as well as detailed comparisons with theoretical models at super-solar metallicities, are required to provide more conclusive evidence for the debate.

Acknowledgements. The authors acknowledge the data analysis facilities provided by the Starlink Project which is run by CCLRC on behalf of PPARC. We thank the referee Dr. P. E. Nissen for many useful suggestions and comments. Support from Fundação para a Ciência e a Tecnologia (Portugal) to N.C.S. in the form of a scholarship (reference SFRH/BPD/8116/2002) and a grant (reference POCI/CTEAST/56453/2004) is gratefully acknowledged.

\section{References}

Abia, C., \& Rebolo, R. 1989, ApJ, 347, 186

Allende Pireto, C., Lambert, D. L., \& Asplund, M. 2001, ApJ, 556, 63
Anders, E., \& Grevesse, N. 1989, Geochim. Cosmochim. Acta, 53, 197

Arnett, W. D. 1978, ApJ, 219, 1008

Asplund, M., Grevesse, N., Sauval, A. J., Allende Prieto, C., \& Kiselman, D. 2004, A\&A, 417, 751

Beirao, P., Santos, N. C., Israelian, G., \& Mayor, M. 2005, A\&A, 438, 251

Belyaev, A. K., Grosser, J., Hahne, J., \& Menzel, T. 1999, Phys. Rev., 60, 2150

Bensby, T., Feltzing, S., \& Lundström, I. 2003, A\&A, 410, 527

Bensby, T., Feltzing, S., \& Lundström, I. 2004, A\&A, 415, 155

Bessell, M. S., Sutherland, R. S., \& Ruan, K. 1991, ApJ, 383, L71

Bodaghee, A., Santos, N. C., Israelian, G., \& Mayor, M. 2003, A\&A, 404, 715

Boesgaard, A. M., King, J. R., Deliyannis, C. P., \& Vogt, S. S. 1999, AJ, 117, 492

Cavallo, R. M., Pilachowski, C. A., \& Rebolo, R. 1997, PASP, 109, 226

Carlsson, M., \& Judge, P. 1993, ApJ, 402, 344

Chiappini, C., Romano, D., \& Matteucci, F. 2003, MNRAS, 339, 63

Drawin, H.-W. 1968, Z. Phys., 211, 404

Ecuvillon, A., Israelian, G., Santos, N. C., et al. 2004a, A\&A, 418, 703

Ecuvillon, A., Israelian, G., Santos, N. C., et al. 2004b, A\&A, 426, $619 \mathrm{E}$

Edvardsson, B., Andersen, J., Gustafsson, B., et al. 1993, A\&A, 275, 101

Feltzing, S., \& Gustafsson, B. 1998, A\&AS, 129, 237

Fulbright, J. P., \& Johnson, J. A. 2003, ApJ, 595, 1154

Gilli, G., Israelian, G., Ecuvillon, A., Santos, N. C., \& Mayor, M. 2005, A\&A, submitted

Gonzalez, G. 1997, MNRAS, 285, 403

Gonzalez, G., \& Laws, C. 2000, AJ, 119, 390

Gonzalez, G., Laws, C., Tyagi, S., \& Reddy, B. E. 2001, AJ, 121, 432

Huber, K. P., \& Herzberg, G. 1979, Constants of Diatomic Molecules (New York: Van Nostrand Reinhold)

Israelian, G. 2004, in IAU S219, Stars as Suns: Activity, Evolution, and Planets, ed. A. K. Dupree (San Francisco: ASP), 219, 343

Israelian, G., García-López, R. J., \& Rebolo, R. 1998, ApJ, 507, 805

Israelian, G., Rebolo, R., García-López, R. J., et al. 2001a, ApJ, 551, 833

Israelian, G., Santos, N. C., Mayor, M., \& Rebolo, R. 2001b, Nature, 411,163

Israelian, G., Santos, N. C., Mayor, M., \& Rebolo, R. 2003, A\&A, 405,753

Israelian, G., Santos, N. C., Mayor, M., \& Rebolo, R. 2004a, A\&A, 414, 601

Israelian, G., Shchukina, N., Rebolo, R., et al. 2004b, A\&A, 419, 1095

King, J. R., \& Boesgaard, A. M. 1995, AJ, 109, 383

Kiselman, D. 1991, A\&A, 245, L9

Kiselman, D. 1993, A\&A, 275, 269

Kiselman, D., \& Nordlund, ̊. 1995, A\&A, 302, 578

Kiselman, D. 2001, New Ast. Rev., 45, 559

Kupka, F. G., Piskunov, N. E., Ryabchikova, T. A., Stempels, H. C., \& Weiss, W. W. 1999, A\&AS, 138, 119

Kurucz, R. L. 1993, CD-ROMs, ATLAS9 Stellar Atmospheres Programs and $2 \mathrm{~km} \mathrm{~s}^{-1}$ Grid (Cambridge: Smithsonian Astrophys. Obs.)

Kurucz, R. L., \& Bell, B. 1995, Kurucz CD-ROM No.23 (HarvardSmithsonian Center for Astrophysics)

Kurucz, R. L., Furenlid, I., Brault, J., \& Testerman, L. 1984, Solar Flux Atlas from 296 to 1300 nm, NOAO Atlas No. 1

Lambert, D. L. 1978, MNRAS, 182, 249 
Laws, C., Gonzalez, G., Walker, K. M., et al. 2003, AJ, 125, 2664

McWilliam, A. 1997, ARA\&A, 35, 503

Mishenina, T., Korotin, S., Klochkova, V., \& Panchuk, V. 2000, A\&A, 353, 978

Nissen, P. E., \& Edvardsson, B. 1992, A\&A, 261, 255

Nissen, P. E., Gustafsson, B., Edvardsson, B., \& Gilmore, G. 1994, A\&A, 285, 440

Nissen, P. E., Primas, F., Asplund, M., \& Lambert, D. L. 2002, A\&A, 390, 235

Pinsonneault, M. H., DePoy, D. L., \& Coffee, M. 2001, ApJ, 556, 59

Prochaska, J. X., Naumov, S. O., Carney, B. W., McWilliam, A., \& Wolfe, A. M. 2000, ApJ, 120, 2513

Sadakane, K., Ohkubo, Y., Takeda, Y., et al. 2002, PASJ, 54, 911

Sandquist, E. L., Dokter, J. J., Lin, D. N. C., \& Mardling, R. 2002, ApJ, 572, 1012

Santos, N. C., Israelian, G., \& Mayor, M. 2000, A\&A, 363, 228

Santos, N. C., Israelian, G., \& Mayor, M. 2001, A\&A, 373, 1019

Santos, N. C., García López, R. J., Israelian, G., et al. 2002, A\&A, 386, 1028

Santos, N. C., Mayor, M., Udry, S., et al., 2003a, in IAU S219, Stars as Suns: Activity, Evolution, and Planets, ed. A. K. Dupree (San Francisco: ASP), 219, 311
Santos, N. C., Israelian, G., Mayor, M., Rebolo, R., \& Udry, S. 2003b, A\&A, 398, 363

Santos, N. C., Israelian, G., \& Mayor, M. 2004a, A\&A, 415, 1153

Santos, N. C., Israelian, G., García López, R. J., et al. 2004b, A\&A, 427,1085

Santos, N. C., Israelian, G., Mayor, M., et al. 2005, A\&A, 437, 1127

Shchukina, N. 1987, Kinemat. Phys. Cel. Bod., 3(6), 36

Shchukina, N. G., Trujillo Bueno, J., \& Asplund, M. 2005, ApJ, 618, 939

Smith, V. V., Cunha, K., \& Lazzaro, D. 2001, AJ, 121, 3207

Sneden, C. 1973, Ph.D. Thesis, University of Texas

Takeda, Y., Sato, B., Kambe, E., et al. 2001, PASJ, 53, 1211

Takeda, Y. 2003, A\&A, 402, 343

Takeda, Y., \& Honda, S. 2005, PASJ, 57, 65

Tinsley, B. M. 1979, ApJ, 229, 1046

Tomkin, J., Lemke, M., Lambert, D. L., \& Sneden, C. 1992, AJ, 104, 1568

Udry, S., Mayor, M., Naef, D., et al. 2000, A\&A, 356, 590

Woosley, S. E., \& Weaver, T. A. 1995, ApJS, 101, 181 
A. Ecuvillon et al.: Oxygen abundances in planet-harbouring stars, Online Material p 1

\section{Online Material}


A. Ecuvillon et al.: Oxygen abundances in planet-harbouring stars, Online Material p 2

Table 4. Oxygen abundances from [OI] line in a set of planet host stars.

\begin{tabular}{|c|c|c|c|c|c|c|c|}
\hline Star & $\begin{array}{l}T_{\text {eff }} \\
(\mathrm{K})\end{array}$ & $\begin{array}{c}\log g \\
\left(\mathrm{~cm} \mathrm{~s}^{-2}\right)\end{array}$ & $\begin{array}{c}\xi_{\mathrm{t}} \\
\left(\mathrm{km} \mathrm{s}^{-1}\right)\end{array}$ & {$[\mathrm{Fe} / \mathrm{H}]$} & $\begin{array}{c}E W_{[\mathrm{O} \text { I }]} \\
(\mathrm{m} \AA)\end{array}$ & {$[\mathrm{O} / \mathrm{H}]_{[\mathrm{O} I]}$} & Instr. \\
\hline HD 1237 & $5536 \pm 50$ & $4.56 \pm 0.12$ & $1.33 \pm 0.06$ & $0.12 \pm 0.06$ & $2.9 \pm 0.5$ & $-0.05 \pm 0.10$ & [1] \\
\hline HD 23079 & $5959 \pm 46$ & $4.35 \pm 0.12$ & $1.20 \pm 0.10$ & $-0.11 \pm 0.06$ & $3.5 \pm 0.5$ & $-0.12 \pm 0.09$ & [1] \\
\hline HD 28185 & $5656 \pm 44$ & $4.45 \pm 0.08$ & $1.01 \pm 0.06$ & $0.22 \pm 0.05$ & $5.4 \pm 0.8$ & $0.22 \pm 0.09$ & [1] \\
\hline HD 30177 & $5591 \pm 50$ & $4.35 \pm 0.12$ & $1.03 \pm 0.06$ & $0.39 \pm 0.06$ & $8.3 \pm 0.5$ & $0.44 \pm 0.07$ & [1] \\
\hline HD 33636 & $6046 \pm 49$ & $4.71 \pm 0.09$ & $1.79 \pm 0.19$ & $-0.08 \pm 0.06$ & $3.2 \pm 0.7$ & $0.02 \pm 0.11$ & [1] \\
\hline HD 37124 & $5546 \pm 30$ & $4.50 \pm 0.03$ & $0.80 \pm 0.07$ & $-0.38 \pm 0.04$ & $4.5 \pm 0.3$ & $-0.07 \pm 0.04$ & [1] \\
\hline HD 39091 & $5991 \pm 27$ & $4.42 \pm 0.10$ & $1.24 \pm 0.04$ & $0.10 \pm 0.04$ & $4.9 \pm 0.5$ & $0.13 \pm 0.07$ & [1] \\
\hline HD 50554 & $6026 \pm 30$ & $4.41 \pm 0.13$ & $1.11 \pm 0.06$ & $0.01 \pm 0.04$ & $4.0 \pm 0.4$ & $0.01 \pm 0.07$ & [1] \\
\hline HD 65216 & $5666 \pm 31$ & $4.53 \pm 0.09$ & $1.06 \pm 0.05$ & $-0.12 \pm 0.04$ & $3.7 \pm 0.5$ & $-0.05 \pm 0.08$ & [1] \\
\hline HD 72659 & $5995 \pm 45$ & $4.30 \pm 0.07$ & $1.42 \pm 0.09$ & $0.03 \pm 0.06$ & $5.5 \pm 0.5$ & $0.11 \pm 0.05$ & [1] \\
\hline HD 73256 & $5518 \pm 49$ & $4.42 \pm 0.12$ & $1.22 \pm 0.06$ & $0.26 \pm 0.06$ & $4.8 \pm 1.0$ & $0.17 \pm 0.12$ & [1] \\
\hline HD 74156 & $6112 \pm 39$ & $4.34 \pm 0.10$ & $1.38 \pm 0.07$ & $0.16 \pm 0.05$ & $4.6 \pm 0.3$ & $0.11 \pm 0.06$ & [1] \\
\hline HD 106252 & $5899 \pm 35$ & $4.34 \pm 0.07$ & $1.08 \pm 0.06$ & $-0.01 \pm 0.05$ & $4.0 \pm 0.8$ & $-0.04 \pm 0.11$ & [1] \\
\hline HD 114729 & $5886 \pm 36$ & $4.28 \pm 0.13$ & $1.25 \pm 0.09$ & $-0.25 \pm 0.05$ & $4.9 \pm 0.5$ & $-0.06 \pm 0.07$ & [1] \\
\hline HD 213240 & $5984 \pm 33$ & $4.25 \pm 0.10$ & $1.25 \pm 0.05$ & $0.17 \pm 0.05$ & $5.7 \pm 0.4$ & $0.15 \pm 0.06$ & [1] \\
\hline HD 216435 & $5938 \pm 42$ & $4.12 \pm 0.05$ & $1.28 \pm 0.06$ & $0.24 \pm 0.05$ & $6.7 \pm 0.7$ & $0.18 \pm 0.06$ & [1] \\
\hline HD 216437 & $5887 \pm 32$ & $4.30 \pm 0.07$ & $1.31 \pm 0.04$ & $0.25 \pm 0.04$ & $6.2 \pm 0.5$ & $0.23 \pm 0.05$ & [1] \\
\hline BD 103166 & $5325 \pm 45$ & $4.36 \pm 0.07$ & $0.95 \pm 0.05$ & $0.35 \pm 0.05$ & $3.9 \pm 1.0$ & $0.06 \pm 0.13$ & [3] \\
\hline HD 2039 & $5976 \pm 51$ & $4.45 \pm 0.10$ & $1.26 \pm 0.07$ & $0.32 \pm 0.06$ & $4.2 \pm 1.0$ & $0.14 \pm 0.12$ & [5] \\
\hline HD 3651 & $5173 \pm 35$ & $4.37 \pm 0.12$ & $0.74 \pm 0.05$ & $0.12 \pm 0.04$ & $5.1 \pm 0.5$ & $0.12 \pm 0.08$ & [4] \\
\hline HD 4203 & $5636 \pm 40$ & $4.23 \pm 0.14$ & $1.12 \pm 0.05$ & $0.40 \pm 0.05$ & $3.8 \pm 1.5$ & $0.00 \pm 0.21$ & [3] \\
\hline HD 9826 & $6212 \pm 64$ & $4.26 \pm 0.13$ & $1.69 \pm 0.16$ & $0.13 \pm 0.08$ & $4.0 \pm 0.7$ & $0.02 \pm 0.10$ & [2] \\
\hline HD 16141 & $5801 \pm 30$ & $4.22 \pm 0.12$ & $1.34 \pm 0.04$ & $0.15 \pm 0.04$ & $4.7 \pm 1.0$ & $0.01 \pm 0.11$ & [3] \\
\hline HD 17051 & $6252 \pm 53$ & $4.61 \pm 0.16$ & $1.18 \pm 0.10$ & $0.26 \pm 0.06$ & $3.6 \pm 0.7$ & $0.16 \pm 0.11$ & [1] \\
\hline HD 19994 & $6190 \pm 00$ & $4.19 \pm 0.00$ & $1.54 \pm 0.00$ & $0.24 \pm 0.00$ & $5.0 \pm 1.0$ & $0.11 \pm 0.09$ & [1] \\
\hline HD 22049 & $5073 \pm 42$ & $4.43 \pm 0.08$ & $1.05 \pm 0.06$ & $-0.13 \pm 0.05$ & $3.8 \pm 0.8$ & $-0.07 \pm 0.10$ & [2] \\
\hline HD 23079 & $5959 \pm 46$ & $4.35 \pm 0.12$ & $1.20 \pm 0.10$ & $-0.11 \pm 0.06$ & $4.2 \pm 0.2$ & $-0.04 \pm 0.06$ & [3] \\
\hline HD 23596 & $6108 \pm 36$ & $4.25 \pm 0.10$ & $1.30 \pm 0.05$ & $0.31 \pm 0.05$ & $5.4 \pm 0.3$ & $0.18 \pm 0.05$ & [2] \\
\hline HD 27442 & $4825 \pm 107$ & $3.55 \pm 0.32$ & $1.18 \pm 0.12$ & $0.39 \pm 0.13$ & $15.6 \pm 1.5$ & $0.36 \pm 0.16$ & [3] \\
\hline HD 28185 & $5656 \pm 44$ & $4.45 \pm 0.08$ & $1.01 \pm 0.06$ & $0.22 \pm 0.05$ & $3.6 \pm 1.0$ & $0.02 \pm 0.15$ & [5] \\
\hline HD 30177 & $5587 \pm 00$ & $4.29 \pm 0.00$ & $1.08 \pm 0.00$ & $0.38 \pm 0.00$ & $3.3 \pm 1.0$ & $-0.04 \pm 0.15$ & [5] \\
\hline HD 38529 & $5674 \pm 40$ & $3.94 \pm 0.12$ & $1.38 \pm 0.05$ & $0.40 \pm 0.06$ & $13.3 \pm 0.7$ & $0.44 \pm 0.06$ & [3] \\
\hline HD 46375 & $5268 \pm 55$ & $4.41 \pm 0.16$ & $0.97 \pm 0.06$ & $0.20 \pm 0.06$ & $5.9 \pm 0.5$ & $0.23 \pm 0.09$ & [2] \\
\hline HD 50554 & $6026 \pm 30$ & $4.41 \pm 0.13$ & $1.11 \pm 0.06$ & $0.01 \pm 0.04$ & $5.3 \pm 0.9$ & $0.14 \pm 0.09$ & [2] \\
\hline HD 52265 & $6105 \pm 00$ & $4.28 \pm 0.00$ & $1.36 \pm 0.00$ & $0.23 \pm 0.00$ & $5.1 \pm 0.7$ & $0.14 \pm 0.05$ & [3] \\
\hline HD 59686 & $4871 \pm 135$ & $3.15 \pm 0.24$ & $1.85 \pm 0.12$ & $0.28 \pm 0.18$ & $33.8 \pm 1.0$ & $0.52 \pm 0.13$ & [3] \\
\hline HD 70642 & $5671 \pm 46$ & $4.39 \pm 0.05$ & $1.01 \pm 0.06$ & $0.20 \pm 0.06$ & $5.5 \pm 0.5$ & $0.17 \pm 0.05$ & [3] \\
\hline HD 73256 & $5518 \pm 49$ & $4.42 \pm 0.12$ & $1.22 \pm 0.06$ & $0.26 \pm 0.06$ & $3.5 \pm 0.9$ & $0.01 \pm 0.14$ & [3] \\
\hline HD 74156 & $6112 \pm 39$ & $4.34 \pm 0.10$ & $1.38 \pm 0.07$ & $0.16 \pm 0.05$ & $7.3 \pm 0.7$ & $0.31 \pm 0.06$ & [3] \\
\hline HD 75289 & $6143 \pm 53$ & $4.42 \pm 0.13$ & $1.53 \pm 0.09$ & $0.28 \pm 0.07$ & $5.6 \pm 0.4$ & $0.14 \pm 0.08$ & [1] \\
\hline HD 75732 & $5279 \pm 62$ & $4.37 \pm 0.18$ & $0.98 \pm 0.07$ & $0.33 \pm 0.07$ & $4.5 \pm 0.7$ & $0.13 \pm 0.11$ & [2] \\
\hline HD 82943 & $6015 \pm 00$ & $4.46 \pm 0.00$ & $1.13 \pm 0.00$ & $0.30 \pm 0.00$ & $4.8 \pm 0.7$ & $0.20 \pm 0.06$ & [1] \\
\hline HD 83443 & $5501 \pm 63$ & $4.46 \pm 0.09$ & $1.07 \pm 0.08$ & $0.39 \pm 0.07$ & $4.4 \pm 1.0$ & $0.17 \pm 0.13$ & [3] \\
\hline HD 92788 & $5758 \pm 37$ & $4.3 \pm 0.04$ & $1.1 \pm 0.04$ & $0.34 \pm 0.05$ & $3.8 \pm 1.0$ & $0.02 \pm 0.13$ & [3] \\
\hline HD 95128 & $5954 \pm 25$ & $4.44 \pm 0.10$ & $1.30 \pm 0.04$ & $0.06 \pm 0.03$ & $5.2 \pm 0.6$ & $0.15 \pm 0.06$ & [4] \\
\hline HD 106252 & $5834 \pm 37$ & $4.22 \pm 0.02$ & $1.06 \pm 0.06$ & $-0.03 \pm 0.05$ & $7.0 \pm 1.0$ & $0.14 \pm 0.07$ & [3] \\
\hline HD 108147 & $6248 \pm 42$ & $4.49 \pm 0.16$ & $1.35 \pm 0.08$ & $0.20 \pm 0.05$ & $3.7 \pm 0.4$ & $0.11 \pm 0.09$ & [1] \\
\hline HD 108874 & $5596 \pm 42$ & $4.37 \pm 0.12$ & $0.89 \pm 0.05$ & $0.23 \pm 0.05$ & $3.0 \pm 0.7$ & $-0.10 \pm 0.12$ & [2] \\
\hline HD 114386 & $4865 \pm 93$ & $4.3 \pm 0.17$ & $0.86 \pm 0.12$ & $-0.04 \pm 0.07$ & $5.1 \pm 1.0$ & $0.07 \pm 0.15$ & [3] \\
\hline HD 114762 & $5884 \pm 34$ & $4.22 \pm 0.02$ & $1.31 \pm 0.17$ & $-0.70 \pm 0.04$ & $3.2 \pm 0.1$ & $-0.36 \pm 0.02$ & [1] \\
\hline HD 114783 & $5098 \pm 36$ & $4.45 \pm 0.11$ & $0.74 \pm 0.05$ & $0.09 \pm 0.04$ & $4.4 \pm 1.5$ & $0.08 \pm 0.18$ & [4] \\
\hline HD 117176 & $5560 \pm 34$ & $4.07 \pm 0.05$ & $1.18 \pm 0.05$ & $-0.06 \pm 0.05$ & $7.1 \pm 0.5$ & $0.05 \pm 0.04$ & [4] \\
\hline HD 121504 & $6075 \pm 40$ & $4.64 \pm 0.12$ & $1.31 \pm 0.07$ & $0.16 \pm 0.05$ & $3.5 \pm 0.2$ & $0.10 \pm 0.06$ & [1] \\
\hline
\end{tabular}


A. Ecuvillon et al.: Oxygen abundances in planet-harbouring stars, Online Material p 3

Table 4. continued.

\begin{tabular}{|c|c|c|c|c|c|c|c|}
\hline Star & $\begin{array}{l}T_{\text {eff }} \\
(\mathrm{K})\end{array}$ & $\begin{array}{c}\log g \\
\left(\mathrm{~cm} \mathrm{~s}^{-2}\right)\end{array}$ & $\begin{array}{c}\xi_{\mathrm{t}} \\
\left(\mathrm{km} \mathrm{s}^{-1}\right)\end{array}$ & {$[\mathrm{Fe} / \mathrm{H}]$} & $\begin{array}{c}E W_{[\mathrm{O} \text { I] }} \\
(\mathrm{m} \AA)\end{array}$ & {$[\mathrm{O} / \mathrm{H}]_{[\mathrm{OI}]}$} & Instr. \\
\hline HD 128311 & $4835 \pm 72$ & $4.44 \pm 0.21$ & $0.89 \pm 0.11$ & $0.03 \pm 0.07$ & $6.0 \pm 1.5$ & $0.19 \pm 0.16$ & [2] \\
\hline HD 134987 & $5776 \pm 29$ & $4.36 \pm 0.07$ & $1.09 \pm 0.04$ & $0.30 \pm 0.04$ & $6.5 \pm 0.5$ & $0.27 \pm 0.05$ & [4] \\
\hline HD 137759 & $4775 \pm 113$ & $3.09 \pm 0.40$ & $1.78 \pm 0.11$ & $0.13 \pm 0.14$ & $34.7 \pm 1.0$ & $0.48 \pm 0.19$ & [4] \\
\hline HD 141937 & $5909 \pm 39$ & $4.51 \pm 0.08$ & $1.13 \pm 0.06$ & $0.10 \pm 0.05$ & $4.6 \pm 0.7$ & $0.13 \pm 0.07$ & [2] \\
\hline HD 143761 & $5853 \pm 25$ & $4.41 \pm 0.15$ & $1.35 \pm 0.07$ & $-0.21 \pm 0.04$ & $4.5 \pm 0.8$ & $-0.02 \pm 0.11$ & [4] \\
\hline HD 145675 & $5311 \pm 87$ & $4.42 \pm 0.18$ & $0.92 \pm 0.10$ & $0.43 \pm 0.08$ & $5.7 \pm 1.0$ & $0.29 \pm 0.12$ & [4] \\
\hline HD 147513 & $5894 \pm 31$ & $4.43 \pm 0.02$ & $1.26 \pm 0.05$ & $0.08 \pm 0.04$ & $5.0 \pm 1.5$ & $0.12 \pm 0.16$ & [3] \\
\hline HD 150706 & $5961 \pm 27$ & $4.50 \pm 0.10$ & $1.11 \pm 0.06$ & $-0.01 \pm 0.04$ & $2.8 \pm 0.5$ & $-0.12 \pm 0.09$ & [2] \\
\hline HD 168443 & $5617 \pm 35$ & $4.22 \pm 0.05$ & $1.21 \pm 0.05$ & $0.06 \pm 0.05$ & $6.3 \pm 1.0$ & $0.11 \pm 0.08$ & [4] \\
\hline HD 168746 & $5601 \pm 33$ & $4.41 \pm 0.12$ & $0.99 \pm 0.05$ & $-0.08 \pm 0.05$ & $6.8 \pm 0.5$ & $0.19 \pm 0.07$ & [4] \\
\hline HD 177830 & $4804 \pm 77$ & $3.57 \pm 0.17$ & $1.14 \pm 0.09$ & $0.33 \pm 0.09$ & $16.0 \pm 0.7$ & $0.38 \pm 0.09$ & [4] \\
\hline HD 178911B & $5600 \pm 42$ & $4.44 \pm 0.08$ & $0.95 \pm 0.05$ & $0.27 \pm 0.05$ & $5.9 \pm 1.0$ & $0.25 \pm 0.08$ & [4] \\
\hline HD 179949 & $6260 \pm 43$ & $4.43 \pm 0.05$ & $1.41 \pm 0.09$ & $0.22 \pm 0.05$ & $2.9 \pm 1.0$ & $-0.02 \pm 0.18$ & [4] \\
\hline HD 186427 & $5772 \pm 25$ & $4.40 \pm 0.07$ & $1.07 \pm 0.04$ & $0.08 \pm 0.04$ & $3.0 \pm 0.3$ & $-0.13 \pm 0.05$ & [4] \\
\hline HD 187123 & $5845 \pm 22$ & $4.42 \pm 0.07$ & $1.10 \pm 0.03$ & $0.13 \pm 0.03$ & $5.6 \pm 0.5$ & $0.18 \pm 0.05$ & [4] \\
\hline HD 190360 & $5584 \pm 36$ & $4.37 \pm 0.06$ & $1.07 \pm 0.05$ & $0.24 \pm 0.05$ & $6.1 \pm 0.6$ & $0.22 \pm 0.04$ & [2] \\
\hline HD 192263 & $4947 \pm 58$ & $4.51 \pm 0.20$ & $0.86 \pm 0.09$ & $-0.02 \pm 0.06$ & $4.1 \pm 1.0$ & $0.04 \pm 0.15$ & [3] \\
\hline HD 195019 & $5842 \pm 31$ & $4.32 \pm 0.07$ & $1.27 \pm 0.05$ & $0.09 \pm 0.04$ & $3.7 \pm 0.7$ & $-0.07 \pm 0.09$ & [2] \\
\hline HD 209458 & $6117 \pm 26$ & $4.48 \pm 0.08$ & $1.40 \pm 0.06$ & $0.02 \pm 0.03$ & $4.0 \pm 0.8$ & $0.06 \pm 0.10$ & [1] \\
\hline HD 210277 & $5532 \pm 00$ & $4.29 \pm 0.00$ & $1.03 \pm 0.00$ & $0.19 \pm 0.00$ & $6.6 \pm 0.5$ & $0.21 \pm 0.04$ & [4] \\
\hline HD 213240 & $5984 \pm 33$ & $4.25 \pm 0.10$ & $1.25 \pm 0.05$ & $0.17 \pm 0.05$ & $9.0 \pm 1.5$ & $0.35 \pm 0.09$ & [5] \\
\hline HD 216770 & $5423 \pm 41$ & $4.40 \pm 0.13$ & $1.01 \pm 0.05$ & $0.26 \pm 0.04$ & $10.2 \pm 2.0$ & $0.49 \pm 0.12$ & [4] \\
\hline HD 217014 & $5804 \pm 36$ & $4.42 \pm 0.07$ & $1.20 \pm 0.05$ & $0.20 \pm 0.05$ & $5.4 \pm 1.0$ & $0.19 \pm 0.11$ & [3] \\
\hline HD 217107 & $5645 \pm 00$ & $4.31 \pm 0.00$ & $1.06 \pm 0.00$ & $0.37 \pm 0.00$ & $5.4 \pm 0.2$ & $0.18 \pm 0.01$ & [2] \\
\hline HD 222582 & $5843 \pm 38$ & $4.45 \pm 0.07$ & $1.03 \pm 0.06$ & $0.05 \pm 0.05$ & $4.8 \pm 1.0$ & $0.10 \pm 0.11$ & [2] \\
\hline HD 104985 & $4773 \pm 62$ & $2.76 \pm 0.14$ & $1.71 \pm 0.07$ & $-0.28 \pm 0.09$ & $42.7 \pm 1.5$ & $0.30 \pm 0.07^{1}$ & [4] \\
\hline
\end{tabular}

The instruments used to obtain the spectra were: [1] UVES; [2] UES; [3] FEROS; [4] SARG; [5] CORALIE.

${ }^{1}$ This value was excluded from the figures since it is inconsistent with the triplet result.

Table 5. Oxygen abundances from $[\mathrm{O} I]$ line in a set of comparison stars.

\begin{tabular}{lccccccc}
\hline \hline Star & $\begin{array}{c}T_{\text {eff }} \\
(\mathrm{K})\end{array}$ & $\begin{array}{c}\log g \\
\left(\mathrm{~cm} \mathrm{~s}^{-2}\right)\end{array}$ & $\begin{array}{c}\xi_{\mathrm{t}} \\
\left(\mathrm{km} \mathrm{s}^{-1}\right)\end{array}$ & {$[\mathrm{Fe} / \mathrm{H}]$} & $\begin{array}{c}E W_{[\mathrm{O} I]} \\
(\mathrm{m} \AA)\end{array}$ & {$[\mathrm{O} / \mathrm{H}]_{[\mathrm{O} I]}$} & Instr. \\
\hline HD 1581 & $5956 \pm 44$ & $4.39 \pm 0.13$ & $1.07 \pm 0.09$ & $-0.14 \pm 0.05$ & $4.8 \pm 0.2$ & $0.03 \pm 0.07$ & {$[3]$} \\
HD 7570 & $6140 \pm 41$ & $4.39 \pm 0.16$ & $1.50 \pm 0.08$ & $0.18 \pm 0.05$ & $3.8 \pm 0.3$ & $0.05 \pm 0.09$ & {$[1]$} \\
HD 52919 & $4740 \pm 49$ & $4.25 \pm 0.1$ & $1.03 \pm 0.09$ & $-0.05 \pm 0.06$ & $4.8 \pm 1.0$ & $-0.04 \pm 0.11$ & {$[3]$} \\
HD 53143 & $5462 \pm 54$ & $4.47 \pm 0.07$ & $1.08 \pm 0.07$ & $0.22 \pm 0.06$ & $1.9 \pm 0.5$ & $-0.25 \pm 0.14$ & {$[3]$} \\
HD 67199 & $5136 \pm 56$ & $4.54 \pm 0.08$ & $0.84 \pm 0.08$ & $0.06 \pm 0.06$ & $6.3 \pm 2.0$ & $0.28 \pm 0.18$ & {$[3]$} \\
HD 100623 & $5246 \pm 37$ & $4.54 \pm 0.05$ & $1 \pm 0.06$ & $-0.38 \pm 0.05$ & $2.3 \pm 0.5$ & $-0.32 \pm 0.10$ & {$[3]$} \\
HD 102365 & $5667 \pm 27$ & $4.59 \pm 0.02$ & $1.05 \pm 0.06$ & $-0.27 \pm 0.04$ & $3.8 \pm 0.5$ & $-0.04 \pm 0.07$ & {$[3]$} \\
HD 104304 & $5562 \pm 50$ & $4.37 \pm 0.06$ & $1.1 \pm 0.06$ & $0.27 \pm 0.06$ & $6.0 \pm 0.5$ & $0.22 \pm 0.05$ & {$[3]$} \\
HD 109200 & $5103 \pm 46$ & $4.47 \pm 0.07$ & $0.75 \pm 0.07$ & $-0.24 \pm 0.05$ & $5.2 \pm 0.4$ & $0.06 \pm 0.05$ & {$[3]$} \\
HD 115617 & $5577 \pm 33$ & $4.34 \pm 0.03$ & $1.07 \pm 0.04$ & $0.01 \pm 0.05$ & $4.6 \pm 1.0$ & $0.00 \pm 0.11$ & {$[3]$} \\
HD 118972 & $5241 \pm 66$ & $4.43 \pm 0.1$ & $1.24 \pm 0.08$ & $-0.01 \pm 0.08$ & $3.6 \pm 0.9$ & $-0.06 \pm 0.19$ & {$[3]$} \\
HD 125072 & $5001 \pm 115$ & $4.39 \pm 0.21$ & $1.21 \pm 0.15$ & $0.24 \pm 0.11$ & $8.3 \pm 1.0$ & $0.39 \pm 0.12$ & {$[3]$} \\
HD 140901 & $5645 \pm 37$ & $4.4 \pm 0.04$ & $1.14 \pm 0.05$ & $0.13 \pm 0.05$ & $6.2 \pm 1.5$ & $0.21 \pm 0.12$ & {$[3]$} \\
HD 144628 & $5071 \pm 43$ & $4.41 \pm 0.06$ & $0.82 \pm 0.07$ & $-0.36 \pm 0.06$ & $6.5 \pm 0.8$ & $0.09 \pm 0.07$ & {$[3]$} \\
HD 146233 & $5786 \pm 35$ & $4.31 \pm 0.03$ & $1.18 \pm 0.05$ & $0.08 \pm 0.05$ & $7.7 \pm 1.0$ & $0.26 \pm 0.07$ & {$[3]$} \\
HD 152391 & $5521 \pm 43$ & $4.54 \pm 0.05$ & $1.29 \pm 0.06$ & $0.03 \pm 0.05$ & $4.7 \pm 0.7$ & $0.12 \pm 0.08$ & {$[3]$} \\
HD 154088 & $5414 \pm 60$ & $4.28 \pm 0.08$ & $1.14 \pm 0.07$ & $0.33 \pm 0.07$ & $6.6 \pm 0.7$ & $0.25 \pm 0.07$ & {$[3]$} \\
HD 156274 & $5300 \pm 32$ & $4.41 \pm 0.04$ & $1 \pm 0.05$ & $-0.33 \pm 0.05$ & $5.0 \pm 0.9$ & $-0.02 \pm 0.09$ & {$[3]$} \\
HD 165499 & $5950 \pm 45$ & $4.31 \pm 0.02$ & $1.14 \pm 0.08$ & $0.01 \pm 0.06$ & $4.5 \pm 1.0$ & $0.01 \pm 0.10$ & {$[3]$} \\
\hline
\end{tabular}


Table 5. continued.

\begin{tabular}{lccccccc}
\hline \hline Star & $\begin{array}{c}T_{\text {eff }} \\
(\mathrm{K})\end{array}$ & $\begin{array}{c}\log g \\
\left(\mathrm{~cm} \mathrm{~s}^{-2}\right)\end{array}$ & $\begin{array}{c}\xi_{\mathrm{t}} \\
\left(\mathrm{km} \mathrm{s}^{-1}\right)\end{array}$ & {$[\mathrm{Fe} / \mathrm{H}]$} & $\begin{array}{c}\left.E W_{[\mathrm{O} I}\right] \\
(\mathrm{m} \AA)\end{array}$ & {$[\mathrm{O} / \mathrm{H}]_{[\mathrm{O} I]}$} & Instr. \\
\hline HD 170657 & $5115 \pm 52$ & $4.48 \pm 0.08$ & $1.13 \pm 0.07$ & $-0.22 \pm 0.06$ & $4.0 \pm 0.5$ & $-0.05 \pm 0.07$ & {$[3]$} \\
HD 172051 & $5634 \pm 30$ & $4.43 \pm 0.03$ & $1.06 \pm 0.05$ & $-0.20 \pm 0.04$ & $4.7 \pm 0.5$ & $-0.01 \pm 0.05$ & {$[3]$} \\
HD 177565 & $5664 \pm 28$ & $4.43 \pm 0.02$ & $1.02 \pm 0.04$ & $0.14 \pm 0.04$ & $6.2 \pm 0.5$ & $0.23 \pm 0.04$ & {$[3]$} \\
HD 192310 & $5069 \pm 49$ & $4.38 \pm 0.19$ & $0.79 \pm 0.07$ & $-0.01 \pm 0.05$ & $4.5 \pm 0.5$ & $0.03 \pm 0.11$ & {$[4]$} \\
HD 222335 & $5260 \pm 41$ & $4.45 \pm 0.11$ & $0.92 \pm 0.06$ & $-0.16 \pm 0.05$ & $8.0 \pm 1.0$ & $0.27 \pm 0.07$ & {$[3]$} \\
\hline
\end{tabular}

The instruments used to obtain the spectra were: [1] UVES; [2] UES; [3] FEROS; [4] SARG; [5] CORALIE.

${ }^{1}$ This value was excluded from the figures since it is inconsistent with the triplet result.

Table 6. Oxygen abundances from $\mathrm{OH}$ band synthesis for a set of stars with planets and brown dwarf companions.

\begin{tabular}{|c|c|c|c|c|c|c|c|c|c|}
\hline Star & $\begin{array}{l}T_{\text {eff }} \\
(\mathrm{K})\end{array}$ & $\begin{array}{c}\log g \\
\left(\mathrm{~cm} \mathrm{~s}^{-2}\right)\end{array}$ & $\begin{array}{c}\xi_{\mathrm{t}} \\
\left(\mathrm{km} \mathrm{s}^{-1}\right)\end{array}$ & {$[\mathrm{Fe} / \mathrm{H}]$} & {$[\mathrm{O} / \mathrm{H}]_{1}$} & {$[\mathrm{O} / \mathrm{H}]_{2}$} & {$[\mathrm{O} / \mathrm{H}]_{3}$} & {$[\mathrm{O} / \mathrm{H}]_{4}$} & {$[\mathrm{O} / \mathrm{H}]_{\mathrm{avg}}$} \\
\hline HD 142 & $6302 \pm 56$ & $4.34 \pm 0.13$ & $1.86 \pm 0.17$ & $0.14 \pm 0.07$ & -0.02 & 0.03 & 0.08 & 0.18 & $0.07 \pm 0.13$ \\
\hline HD 1237 & $5536 \pm 50$ & $4.56 \pm 0.12$ & $1.33 \pm$ & $0.12 \pm 0.06$ & 0.06 & 0.01 & 0.06 & 0.16 & 0.10 \\
\hline HD 4208 & $5626 \pm 32$ & $4.49 \pm 0.10$ & $0.95 \pm 0.06$ & $-0.24 \pm 0.04$ & -0.15 & -0.10 & -0.20 & -0.10 & $-0.14 \pm 0.08$ \\
\hline HD 23079 & $5959 \pm 46$ & $4.35 \pm 0.12$ & $1.20 \pm 0.10$ & $-0.11 \pm 0.06$ & -0.02 & 0.03 & 0.03 & 0.03 & $0.02 \pm 0.09$ \\
\hline HD 28185 & $5656 \pm 44$ & $4.45 \pm 0.08$ & $1.01 \pm 0.06$ & $0.22 \pm 0.05$ & 0.16 & 0.26 & 0.21 & 0.31 & $0.23 \pm 0.10$ \\
\hline HD 30177 & $5591 \pm 50$ & $4.35 \pm 0.12$ & $1.03 \pm 0.06$ & $0.39 \pm 0.06$ & 0.23 & 0.23 & 0.38 & 0.38 & $0.31 \pm 0.12$ \\
\hline HD 33636 & $6046 \pm 49$ & $4.71 \pm 0.09$ & $1.79 \pm 0.19$ & $-0.08 \pm 0.06$ & 0.01 & 0.06 & 0.11 & 0.11 & $0.07 \pm 0.10$ \\
\hline HD 37124 & $5546 \pm 30$ & $4.50 \pm 0.03$ & $0.80 \pm 0.07$ & $-0.38 \pm 0.04$ & -0.14 & -0.04 & -0.04 & -0.04 & $-0.06 \pm 0.08$ \\
\hline HD 39091 & $5991 \pm 27$ & $4.42 \pm 0.10$ & $1.24 \pm 0.04$ & $0.10 \pm 0.04$ & 0.19 & 0.19 & 0.24 & 0.29 & $0.23 \pm 0.08$ \\
\hline HD 47536 & $4554 \pm 85$ & $2.48 \pm 0.23$ & $1.82 \pm 0.08$ & $-0.54 \pm 0.12$ & -0.30 & -0.30 & -0.20 & -0.35 & $-0.29 \pm 0.12$ \\
\hline HD 50554 & $6026 \pm 30$ & $4.41 \pm 0.13$ & $1.11 \pm 0.06$ & $0.01 \pm 0.04$ & 0.15 & 0.15 & 0.15 & 0.25 & $0.17 \pm 0.09$ \\
\hline HD 5 & $4871 \pm 135$ & $3.15 \pm 0.41$ & $1.85 \pm 0.12$ & $0.28 \pm 0.18$ & 0.12 & 0.12 & 0.22 & 0.12 & $0.14 \pm 0.16$ \\
\hline HD 65216 & $5666 \pm 31$ & $4.53 \pm 0.09$ & $1.06 \pm 0.05$ & $-0.12 \pm 0.04$ & -0.08 & 0.02 & 0.02 & 0.02 & $-0.00 \pm 0.08$ \\
\hline $\mathrm{HD} 7$ & $5693 \pm 26$ & $4.41 \pm 0.09$ & $1.01 \pm 0.04$ & $0.18 \pm 0.04$ & 0.12 & 0.12 & 0.22 & 0.22 & $0.17 \pm 0.09$ \\
\hline HD 72659 & $5995 \pm 45$ & $4.30 \pm 0.07$ & $1.42 \pm 0.09$ & $0.03 \pm 0.06$ & 0.17 & 0.17 & 0.22 & 0.27 & $0.21 \pm 0.09$ \\
\hline HD 73256 & $5518 \pm 49$ & $4.42 \pm 0.12$ & $1.22 \pm 0.06$ & $0.26 \pm 0.06$ & 0.00 & 0.10 & 0.15 & 0.20 & $0.11 \pm 0.11$ \\
\hline HD 74156 & $6112 \pm 39$ & $4.34 \pm 0.10$ & $1.38 \pm 0.07$ & $0.16 \pm 0.05$ & 0.30 & 0.30 & 0.35 & 0.35 & $0.32 \pm 0.08$ \\
\hline HD 8 & $5438 \pm 34$ & $3.94 \pm 0.11$ & $1.16 \pm 0.03$ & $0.33 \pm 0.05$ & 0.17 & 0.17 & 0.27 & 0.27 & $0.22 \pm 0.09$ \\
\hline HD 99492 & & $4.21 \pm 0.21$ & & & 0.00 & & & & 0.08 \\
\hline HD 106252 & $5899 \pm 35$ & $4.34 \pm 0.07$ & $1.08 \pm 0.06$ & $-0.01 \pm 0.05$ & 0.08 & 0.08 & 0.13 & 0.08 & $0.09 \pm 0.07$ \\
\hline HD 114729 & & $4.28 \pm 0.13$ & $1.25 \pm 0.09$ & $-0.25 \pm 0.05$ & -0.01 & -0.01 & -0.01 & -0.01 & 0.07 \\
\hline HD 117207 & $5654 \pm 33$ & $4.32 \pm 0.05$ & $1.13 \pm 0.04$ & $0.23 \pm 0.05$ & 0.12 & 0.17 & 0.22 & 0.27 & $0.19 \pm 0.09$ \\
\hline HD & & $4.39 \pm 0.07$ & $1.73 \pm 0.09$ & & 0.12 & & & & 0.09 \\
\hline HD 213240 & $5984 \pm 33$ & $4.25 \pm 0.10$ & $1.25 \pm 0.05$ & $0.17 \pm 0.05$ & 0.23 & 0.21 & 0.26 & 0.31 & $0.25 \pm 0.08$ \\
\hline HD 2 & & $4.12 \pm 0.05$ & $1.28 \pm 0.06$ & $0.24 \pm 0.05$ & 0.20 & & & 33 & 0.12 \\
\hline HD 216437 & $5887 \pm 32$ & $4.30 \pm 0.07$ & $1.31 \pm 0.04$ & $0.25 \pm 0.04$ & 0.20 & 0.13 & 0.28 & 0.28 & $0.22 \pm 0.10$ \\
\hline HD 219449 & $4757 \pm 102$ & $2.71 \pm 0.25$ & $1.71 \pm 0.09$ & $0.05 \pm 0.14$ & -0.16 & -0.11 & -0.01 & -0.11 & -0.10 \\
\hline HD 6434 & $5835 \pm 50$ & $4.60 \pm 0.15$ & $1.53 \pm 0.10$ & $-0.52 \pm 0.05$ & -0.26 & -0.13 & -0.20 & -0.13 & $-0.18 \pm 0.10$ \\
\hline HD 9826 & $6212 \pm 64$ & $4.26 \pm 0.13$ & $1.69 \pm 0.16$ & $0.13 \pm 0.08$ & 0.17 & 0.27 & 0.17 & 0.27 & $0.22 \pm 0.12$ \\
\hline HD 10647 & $6143 \pm 31$ & $4.48 \pm 0.08$ & $1.40 \pm 0.08$ & $-0.03 \pm 0.04$ & -0.01 & -0.11 & -0.01 & -0.11 & $-0.06 \pm 0.09$ \\
\hline HD 13445 & $5163 \pm 37$ & $4.52 \pm 0.13$ & $0.72 \pm 0.06$ & $-0.24 \pm 0.05$ & -0.40 & -0.20 & -0.20 & -0.20 & $-0.25 \pm 0.12$ \\
\hline HD 16141 & $5801 \pm 30$ & $4.22 \pm 0.12$ & $1.34 \pm 0.04$ & $0.15 \pm 0.04$ & 0.09 & 0.24 & 0.25 & 0.20 & $0.19 \pm 0.10$ \\
\hline HD 17051 & $6252 \pm 53$ & $4.61 \pm 0.16$ & $1.18 \pm 0.10$ & $0.26 \pm 0.06$ & 0.29 & 0.34 & 0.39 & 0.29 & $0.33 \pm 0.11$ \\
\hline HD 19994 & $6190 \pm 57$ & $4.19 \pm 0.13$ & $1.54 \pm 0.13$ & $0.24 \pm 0.07$ & 0.28 & 0.33 & 0.38 & 0.23 & $0.31 \pm 0.12$ \\
\hline HD 22049 & $5073 \pm 42$ & $4.43 \pm 0.08$ & $1.05 \pm 0.06$ & $-0.13 \pm 0.04$ & -0.34 & -0.18 & -0.22 & -0.19 & $-0.23 \pm 0.10$ \\
\hline HD 27442 & $4825 \pm 107$ & $3.55 \pm 0.32$ & $1.18 \pm 0.12$ & $0.39 \pm 0.13$ & -0.02 & 0.16 & 0.08 & - & $0.07 \pm 0.14$ \\
\hline HD 46375 & $5268 \pm 55$ & $4.41 \pm 0.16$ & $0.97 \pm 0.06$ & $0.20 \pm 0.06$ & -0.06 & 0.09 & 0.09 & 0.04 & $0.04 \pm 0.10$ \\
\hline HD 52265 & $6103 \pm 52$ & $4.28 \pm 0.15$ & $1.36 \pm 0.09$ & $0.23 \pm 0.07$ & 0.19 & 0.19 & 0.27 & 0.19 & $0.21 \pm 0.10$ \\
\hline HD 75289 & $6143 \pm 53$ & $4.42 \pm 0.13$ & $1.53 \pm 0.09$ & $0.28 \pm 0.07$ & 0.19 & 0.19 & 0.29 & 0.19 & $0.22 \pm 0.11$ \\
\hline HD 82943 & $6016 \pm 30$ & $4.46 \pm 0.08$ & $1.13 \pm 0.04$ & $0.30 \pm 0.04$ & 0.24 & 0.29 & 0.39 & 0.24 & $0.29 \pm 0.10$ \\
\hline
\end{tabular}


A. Ecuvillon et al.: Oxygen abundances in planet-harbouring stars, Online Material p 5

Table 6. continued.

\begin{tabular}{|c|c|c|c|c|c|c|c|c|c|}
\hline Star & $\begin{array}{l}T_{\text {eff }} \\
(\mathrm{K})\end{array}$ & $\begin{array}{c}\log g \\
\left(\mathrm{~cm} \mathrm{~s}^{-2}\right)\end{array}$ & $\begin{array}{c}\xi_{\mathrm{t}} \\
\left(\mathrm{km} \mathrm{s}^{-1}\right)\end{array}$ & {$[\mathrm{Fe} / \mathrm{H}]$} & {$[\mathrm{O} / \mathrm{H}]_{1}$} & {$[\mathrm{O} / \mathrm{H}]_{2}$} & {$[\mathrm{O} / \mathrm{H}]_{3}$} & {$[\mathrm{O} / \mathrm{H}]_{4}$} & {$[\mathrm{O} / \mathrm{H}]_{\mathrm{avg}}$} \\
\hline HD 8 & 1 & $33 \pm 0.17$ & $1.08 \pm 0.08$ & $0.35 \pm 0.08$ & -0.01 & 0.19 & 0.19 & - & $0.12 \pm 0.13$ \\
\hline HD 143761 & $5853 \pm 25$ & $4.41 \pm 0.15$ & $1.35 \pm 0.07$ & $-0.21 \pm 0.04$ & -0.17 & -0.07 & -0.07 & -0.07 & $-0.09 \pm 0.08$ \\
\hline HD 169830 & $6299 \pm 41$ & $4.10 \pm 0.02$ & $1.42 \pm 0.09$ & $0.21 \pm 0.05$ & 0.19 & 0.29 & 0.29 & 0.09 & $0.22 \pm 0.12$ \\
\hline HD 179949 & $6260 \pm 43$ & $4.43 \pm 0.05$ & $1.41 \pm 0.09$ & $0.22 \pm 0.05$ & 0.22 & 0.34 & 0.29 & 0.19 & $0.26 \pm 0.11$ \\
\hline HD 192263 & $4947 \pm 58$ & $4.51 \pm 0.20$ & $0.86 \pm 0.09$ & $-0.02 \pm 0.06$ & -0.21 & -0.11 & -0.18 & -0.16 & $-0.17 \pm 0.09$ \\
\hline HD 202206 & $5752 \pm 53$ & $4.50 \pm 0.09$ & $1.01 \pm 0.06$ & $0.35 \pm 0.06$ & 0.14 & 0.24 & 0.24 & 0.19 & $0.20 \pm 0.09$ \\
\hline HD 209458 & $6117 \pm 26$ & $4.48 \pm 0.08$ & $1.40 \pm 0.06$ & $0.02 \pm 0.03$ & -0.01 & 0.06 & 0.06 & 0.02 & $0.03 \pm 0.07$ \\
\hline HD 210277 & $5532 \pm 28$ & $4.29 \pm 0.09$ & $1.04 \pm 0.03$ & $0.19 \pm 0.04$ & -0.01 & 0.19 & 0.22 & 0.11 & $0.13 \pm 0.12$ \\
\hline HD 217014 & $5804 \pm 36$ & $4.42 \pm 0.07$ & $1.20 \pm 0.05$ & $0.20 \pm 0.05$ & 0.04 & -0.11 & -0.01 & -0.16 & $-0.06 \pm 0.11$ \\
\hline HD 217107 & $5646 \pm 34$ & $4.31 \pm 0.10$ & $1.06 \pm 0.04$ & $0.37 \pm 0.05$ & 0.14 & 0.34 & 0.39 & 0.29 & $0.29 \pm 0.13$ \\
\hline HD 222582 & $5843 \pm 38$ & $4.45 \pm 0.07$ & $1.03 \pm 0.06$ & $0.05 \pm 0.05$ & 0.04 & 0.14 & 0.09 & 0.09 & $0.09 \pm 0.08$ \\
\hline
\end{tabular}

Table 7. Oxygen abundances from $\mathrm{OH}$ band synthesis for a set of comparison stars (stars without giant planets).

\begin{tabular}{|c|c|c|c|c|c|c|c|c|c|}
\hline Star & $\begin{array}{l}T_{\text {eff }} \\
(\mathrm{K})\end{array}$ & $\begin{array}{c}\log g \\
\left(\mathrm{~cm} \mathrm{~s}^{-2}\right)\end{array}$ & $\begin{array}{c}\xi_{\mathrm{t}} \\
\left(\mathrm{km} \mathrm{s}^{-1}\right)\end{array}$ & H] & $/ \mathrm{H}]_{1}$ & & & 4 & I $]_{\text {avg }}$ \\
\hline & & & & & & & & & \\
\hline & & & & & & & & & \\
\hline P & & & & 0 & & & & & \\
\hline & & & & & & & & & \\
\hline & & $4.39=$ & & & & & & & \\
\hline & & & & & & & & & \\
\hline HD & & 4 & & & & & & & \\
\hline & & & & & & & & & \\
\hline HD & & & & -0 & & & & & .08 \\
\hline & & & & & & & & & \\
\hline & & & & 4 & & & & & .07 \\
\hline & & & & & & & & & \\
\hline & & & & & & & & & .09 \\
\hline & & & & & & & & & \\
\hline & & & & & & & & & .08 \\
\hline & & & & & & & & & .09 \\
\hline & & & & & & & & & \\
\hline & & & & & & & & & .09 \\
\hline & & & & & & & & & \\
\hline & 5 & 4.5 & & -0 . & -0 & & & & .10 \\
\hline & & & & & & & & & \\
\hline & & & $1.42 \pm$ & -0 & & & & & .09 \\
\hline & & & $1.22 \pm$ & & & & & & .09 \\
\hline & & & $0.79 \pm$ & -0.0 & & & & & 0.11 \\
\hline & & & & & & & & & \\
\hline HD 222335 & $5260 \pm 41$ & $4.45 \pm 0.11$ & $0.92 \pm 0.06$ & $-0.16 \pm 0.05$ & -0.32 & -0.17 & -0.10 & -0.17 & $-0.19 \pm 0.12$ \\
\hline
\end{tabular}


A. Ecuvillon et al.: Oxygen abundances in planet-harbouring stars, Online Material p 6

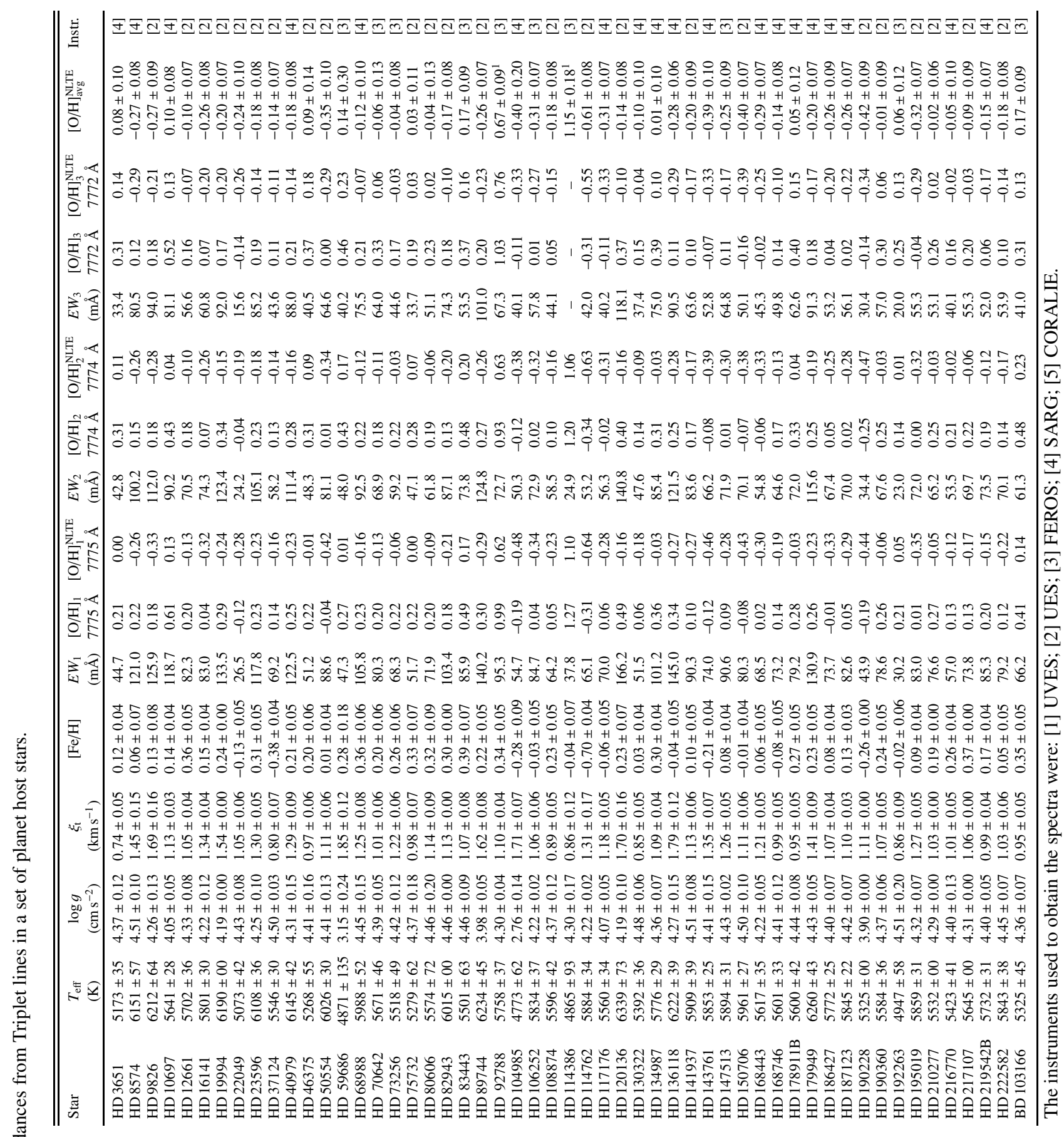


A. Ecuvillon et al.: Oxygen abundances in planet-harbouring stars, Online Material p 7

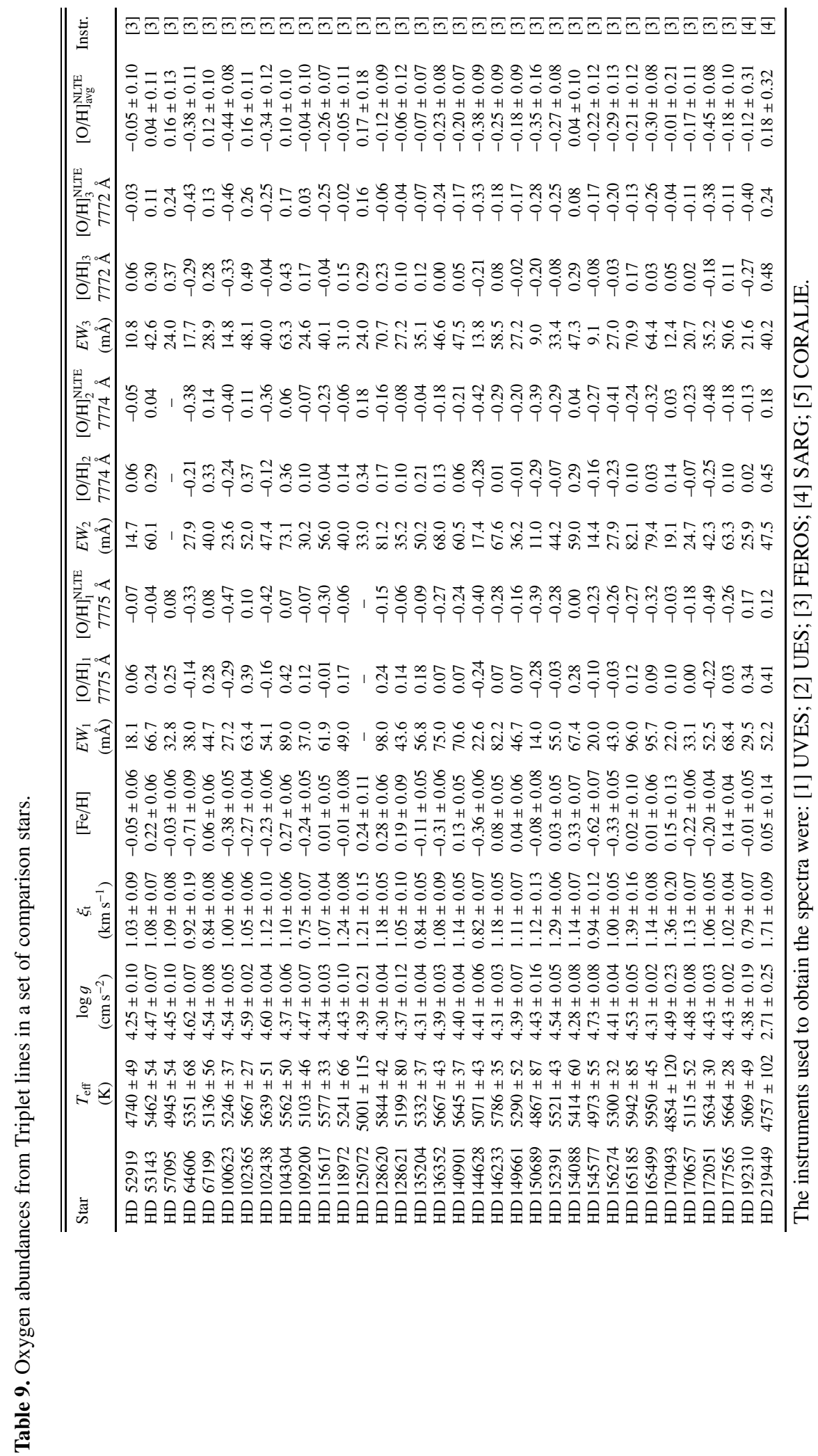

